\title{
Fixed Points and Limits of Convolution Powers of Contractive Quantum Measures
}

\author{
Matthias Neufang, PeKKa Salmi, \\ ADAM SKALSKI \& NICO SPRONK
}

\begin{abstract}
We study fixed points of contractive convolution operators associated with contractive quantum measures on locally compact quantum groups. We characterise the existence of non-zero fixed points, respectively, on $L^{\infty}(\mathbb{G})$ and on $C_{0}(\mathbb{G})$, and exploit these results to obtain, for example, the structure of the fixed points on the non-commutative $L_{p}$-spaces. Some consequences for the fixed points of classical convolution operators and Herz-Schur multipliers are also indicated.
\end{abstract}

Convolution operators associated with measures on a locally compact group $G$ form a rich and interesting class of transformations, acting on various function spaces associated with $G$, and playing a key role in the study of probabilistic, geometric, and harmonic-analytic phenomena related to $G$. In particular, the random walk interpretations provide motivation to analyse the limit behaviour of iterations of convolution operators (as nicely described in [Grn] and presented from the point of view of quantum generalisations in [Sal]), and thus in particular the nature of idempotent probability measures. The latter are very well understood, but, perhaps surprisingly, dropping the positivity requirement makes the problem of characterising the class of idempotent measures very difficult, and it is only fully solved for contractive measures [Gre] and for abelian groups [Coh]. More generally, one can ask about the structure of the space of fixed points of a given convolution operator (see [ChL], of which more will be said below), which at least in the positive case can be interpreted as the collection of harmonic functions for a given measure- or as the space of functions on the corresponding Poisson boundary (see, e.g., [Kai] and references therein). 
If the group $G$ in question is abelian, the convolution operators can be also viewed as Fourier multipliers. This perspective makes it natural to study also fixed points of such operators even when $G$ is non-abelian. This is to a large extent the point of view taken in the lecture notes [ChL], where a lot of care is devoted to studying, for example, the space of fixed points of contractive HerzSchur multipliers acting on the group von Neumann algebra $\operatorname{VN}(G)$.

The theory of locally compact quantum groups, as formulated by Kustermans and Vaes in $[\mathrm{KV}]$, provides a framework which allows asking these types of questions from a unified perspective, at the same time vastly generalising the class of objects studied. The concept of quantum convolution operators (as investigated in, e.g., [JNR] and [Daw]) has now been reasonably well understood, and in particular several questions related to the nature of idempotent states $\left(\left[\mathrm{SaS}_{1}\right]\right.$ and references therein) or the structure of the fixed points of positive quantum convolution operators $\left(\left[\mathrm{KNR}_{1}\right],\left[\mathrm{KNR}_{2}\right]\right)$ have found satisfactory answers. In our previous work [NSSS] we looked at the idempotent contractive quantum measures and characterised their form, generalising the results of Greenleaf mentioned above (see also [Kas]). Here, we investigate the structure of the fixed point spaces, so in other words "generalised harmonic elements," associated with arbitrary quantum contractive measures.

The quantum context necessitates a distinction between the "universal" and "reduced" quantum measures (respectively, understood as bounded functionals on the universal and reduced algebra of continuous functions on $\mathbb{G}$ vanishing at infinity); both of these induce convolution operators on the algebra $L^{\infty}(\mathbb{G})$ (as well as, e.g., on $C_{0}(\mathbb{G})$ ). We work primarily in the more general setting of universal measures. It is natural to expect that the space of fixed points of a given operator is related to a limit of its Cesáro averages. To be able to exploit this fully, we introduce yet another class of generalised measures, that is, the dual of the space of "universal right uniformly continuous functions." With this in hand, we are able to show our first main result, Theorem 2.3, characterising the existence of non-zero fixed points for the convolution operator on $L^{\infty}(\mathbb{G})$. The results on the structure of the fixed point space, often showing that it is in fact one dimensional (if the measure in question is non-degenerate) require assuming more about the nature of a potential fixed point. The central theorems of this type are Theorem 2.7 and Proposition 4.3. These results, although at first glance quite technical in nature, turn out to have several interesting consequences, both in the general quantum group context (where, e.g., they allow us to characterise fixed points in non-commutative $L_{p}$-spaces), but also in the classical framework and its dual, where they lead to generalisations of several earlier theorems (e.g., of [ChL]). In several instances, we are able to connect the fixed points of the convolution by a quantum contractive measure $\omega$ to the fixed points of the convolution by the absolute value of $\omega$, which opens the way to exploiting the state results already available in the literature.

Finally, we would like to recall that the fixed points of a positive (quantum) convolution operator admit a canonical structure of a von Neumann algebra 
equipped with the action of the (quantum) group in question; this is nothing but a (quantum) Poisson boundary, as mentioned above. Once we consider a general contractive (quantum) convolution operator, the fixed-point space admits a canonical TRO structure, and the corresponding (quantum) group action. The study of such actions was initiated in $\left[\mathrm{SaS}_{2}\right]$; it is, however, fair to say that it remains in a relatively early stage, and deeper harmonic analysis applications are yet to be developed.

The detailed plan of the paper is as follows. In Section 1, we recall basic facts and notation related to locally compact quantum groups, and introduce the algebra $\mathrm{RUC}^{u}(\mathbb{G})^{*}$ and associated quantum convolution operators, which play a useful technical role in what follows. In Section 2, non-zero fixed points in $L^{\infty}(\mathbb{G})$ are studied, and their existence characterised. Here also we get the first results on the structure of the fixed point space if the "universal" convolution operator admits a non-zero fixed point in $\operatorname{LUC}^{u}(\mathbb{G})$. In Section 3, we turn our attention to the preduals of $L^{\infty}(\mathbb{G})$-fixed points, and use them to characterise certain properties of the quantum group in question. The fourth section concerns fixed points in $C_{0}(\mathbb{G})$; the results obtained there are used in the fifth section to discuss the existence of non-zero fixed points in $L_{p}(\mathbb{G})$ (for tracial Haar weights). In Section 6, the general results are specialised to the context of classical locally compact groups, and appropriately strengthened. Finally, in Section 7 we discuss the "dual to classical" case.

\section{Preliminaries}

1.1. Locally compact quantum groups. We follow here the von Neumann algebraic approach to locally compact quantum groups due to Kustermans and Vaes $[\mathrm{KV}]$ (see also $\left[\mathrm{KNR}_{1}\right]$ and $\left[\mathrm{KNR}_{2}\right]$ for more background). A locally compact quantum group $\mathbb{G}$, effectively a virtual object, is studied via the von Neumann algebra $L^{\infty}(\mathbb{G})$, playing the role of the algebra of essentially bounded functions on $\mathbb{G}$, equipped with a comultiplication $\Delta: L^{\infty}(\mathbb{G}) \rightarrow L^{\infty}(\mathbb{G}) \bar{\otimes} L^{\infty}(\mathbb{G})$, which is a unital normal coassociative $*$-homomorphism. A locally compact quantum group $\mathbb{G}$ is by definition assumed to admit a left Haar weight $\phi$ and a right Haar weight $\psi$-these are faithful, normal semifinite weights on $L^{\infty}(\mathbb{G})$ satisfying suitable invariance conditions. We can associate with $\mathbb{G}$ also its algebra of continuous functions vanishing at infinity, $C_{0}(\mathbb{G})$. It is a $C^{*}$-subalgebra of $L^{\infty}(\mathbb{G})$, and the comultiplication has a restriction to a map from $C_{0}(\mathbb{G})$ to the multiplier algebra of $C_{0}(\mathbb{G}) \otimes C_{0}(\mathbb{G})$. We say that $\mathbb{G}$ is compact if $C_{0}(\mathbb{G})$ is unital, in which case we denote it simply $C(\mathbb{G})$. The GNS representation space for the left Haar weight will be denoted by $L^{2}(\mathbb{G})$. We may in fact assume that $C_{0}(\mathbb{G})$ is a non-degenerate subalgebra of $B\left(L^{2}(\mathbb{G})\right)$. Each locally compact quantum group $\mathbb{G}$ admits the dual locally compact quantum group $\hat{\mathbb{G}}$; in fact, the algebra $L^{\infty}(\hat{\mathbb{G}})$ acts naturally on $L^{2}(\mathbb{G})$. We call $\mathbb{G}$ discrete, if $\hat{\mathbb{G}}$ is compact. If $\mathbb{G}=G$ happens to be a locally compact group, then $L^{\infty}(\hat{\mathbb{G}})=\mathrm{VN}(G)$. Finally, note that by analogy with the classical situation, we denote the predual of $L^{\infty}(\mathbb{G})$ by $L^{1}(\mathbb{G})$. 
With each locally compact quantum group $\mathbb{G}$, we may also associate a universal $C^{*}$-algebra $C_{0}^{u}(\mathbb{G})$ with comultiplication $\Delta_{u}$ (see [Kus]). There is a surjective $*$-homomorphism $\Lambda: C_{0}^{u}(\mathbb{G}) \rightarrow C_{0}(\mathbb{G})$ such that $(\Lambda \otimes \Lambda) \circ \Delta_{u}=\Delta \circ \Lambda$. We call $\Lambda$ the reducing morphism of $\mathbb{G}$. The reducing morphism of the dual $\hat{\mathbb{G}}$ is denoted by $\hat{\Lambda}: C_{0}^{u}(\hat{\mathbb{G}}) \rightarrow C_{0}(\hat{\mathbb{G}})$.

The comultiplication $\Delta$ of $C_{0}(\mathbb{G})$ is implemented by the multiplicative unitary $\mathrm{W} \in M\left(C_{0}(\mathbb{G}) \otimes C_{0}(\hat{\mathbb{G}})\right):$

$$
\Delta(x)=\mathrm{W}^{*}(1 \otimes x) \mathrm{W}, \quad x \in C_{0}(\mathbb{G}) .
$$

The multiplicative unitary $\mathrm{W}$ admits a universal lift $\mathbb{W} \in M\left(C_{0}^{u}(\mathbb{G}) \otimes C_{0}^{u}(\hat{\mathbb{G}})\right)$ such that $(\Lambda \otimes \hat{\Lambda})(\mathbb{W})=W$. We shall also need half-universal versions of the bicharacter and the comultiplication: define $\mathbb{W}=(\operatorname{id} \otimes \hat{\Lambda}) \mathbb{W}$.

The unitary antipode of $\mathbb{G}$ will be denoted by $R$. It is a *-antiautomorphism of $C_{0}(\mathbb{G})$ (and, in fact, of $B\left(L^{2}(\mathbb{G})\right)$ ) of order 2. Its universal version, which is a ${ }^{*}$-antiautomorphism of $C_{0}^{u}(\mathbb{G})$ of order 2 , will be denoted by $R_{u}$. We have $\Lambda \circ R_{u}=R \circ \Lambda$.

We shall often write $M^{u}(\mathbb{G}):=C_{0}^{u}(\mathbb{G})^{*}$ and $M(\mathbb{G}):=C_{0}(\mathbb{G})^{*}$, and denote their unit balls by $M^{u}(\mathbb{G})_{1}$ and $M(\mathbb{G})_{1}$, respectively. Note that $M^{u}(\mathbb{G})$ is a completely contractive Banach algebra with respect to convolution $\star$, defined as the adjoint of comultiplication $\Delta_{u}$. Similarly, $M(\mathbb{G})$ is also a completely contractive Banach algebra. define

1.2. Convolution operators. Fix a locally compact quantum group $\mathbb{G}$ and

$$
\Delta_{S}(x)=\mathbb{W}^{*}(1 \otimes x) \mathbb{W}, \quad x \in L^{\infty}(\mathbb{G}) .
$$

Note that $\mathbb{W}$ as well as $\Delta_{S}(x)$ are in $M\left(C_{0}^{u}(\mathbb{G}) \otimes \mathcal{K}\left(L^{2}(\mathbb{G})\right)\right)$ [Kus]. Moreover, by equation (6.1) of [Kus] (applied to W), we have that

$$
\mathbb{W}^{*}(1 \otimes \tilde{\Lambda}(y)) \mathbb{W}=(\mathrm{id} \otimes \tilde{\Lambda}) \circ \Delta_{u}(y), \quad y \in C_{0}^{u}(\mathbb{G})^{* *},
$$

where $\tilde{\Lambda}: C_{0}^{u}(\mathbb{G})^{* *} \rightarrow L^{\infty}(\mathbb{G})$ is the normal extension of the reducing morphism $C_{0}^{u}(\mathbb{G}) \rightarrow C_{0}(\mathbb{G})$. that

For every $\omega \in \mathrm{M}^{u}(\mathbb{G})$ and $\phi \in L^{1}(\mathbb{G})$, there is a unique $\psi \in L^{1}(\mathbb{G})$ such

$$
(\omega \otimes \phi \Lambda) \Delta_{u}(a)=(\psi \Lambda)(a), \quad a \in C_{0}^{u}(\mathbb{G}) .
$$

We will write $\omega \star \phi:=\psi$. Similarly, $\phi \star \omega \in L^{1}(\mathbb{G})$ is the unique $\psi^{\prime} \in L^{1}(\mathbb{G})$ such that

$$
(\phi \Lambda \otimes \omega) \Delta_{\mathcal{u}}(a)=\left(\psi^{\prime} \Lambda\right)(a), \quad a \in C_{0}^{u}(\mathbb{G}) .
$$


Hence, we consider $L^{1}(\mathbb{G})$ an ideal in $M^{u}(\mathbb{G})$. Note that for $x \in L^{\infty}(\mathbb{G})$ we have $\omega \star \phi(x)=\omega\left((\operatorname{id} \otimes \phi) \Delta_{S}(x)\right)$. We will also need the following formula:

$$
(\omega \star \phi) \circ R=(\phi \circ R) \star\left(\omega \circ R_{u}\right), \quad \omega \in \mathrm{M}^{u}(\mathbb{G}), \phi \in L^{1}(\mathbb{G}) .
$$

This follows from the above characterisations of the respective convolutions and the equality $\chi\left(R_{u} \otimes R_{u}\right) \circ \Delta_{u}=\Delta_{u} \circ R_{u}$ established in [Kus].

Let

$$
\operatorname{RUC}^{u}(\mathbb{G})=\overline{\operatorname{span}}\left\{(\mathrm{id} \otimes \phi) \Delta_{S}(x) \mid \phi \in L^{1}(\mathbb{G}), x \in L^{\infty}(\mathbb{G})\right\} .
$$

This is the universal version of RUC $(\mathbb{G})$ introduced and studied in [Run]; we have $\Lambda\left(\operatorname{RUC}^{u}(\mathbb{G})\right)=\operatorname{RUC}(\mathbb{G})$.

Lemma 1.1. The following hold:

(i) $C_{0}^{u}(\mathbb{G})=\overline{\operatorname{span}}\left\{(\mathrm{id} \otimes \phi) \Delta_{s}(a) \mid \phi \in L^{1}(\mathbb{G}), a \in C_{0}(\mathbb{G})\right\}$.

(ii) $\operatorname{RUC}^{u}(\mathbb{G})$ is an operator system, (i.e., a unital and self-adjoint linear closed subspace) in $M\left(C_{0}^{u}(\mathbb{G})\right)$.

Proof. (i) The elements of the form (id $\otimes \sigma) \mathrm{W}$, with $\sigma \in B\left(L^{2}(\mathbb{G})\right)_{*}$, are dense in $C_{0}(\mathbb{G})$, and similarly, by equation (5.2) of [Kus], the elements of the form (id $\otimes \sigma) \mathbb{W}$, with $\sigma \in B\left(L^{2}(\mathbb{G})\right)_{*}$, are dense in $C_{0}^{u}(\mathbb{G})$. By (1.1) and Proposition 6.1 of [Kus], we have

$$
\left(\Delta_{S} \otimes \mathrm{id}\right)(\mathrm{W})=(\mathrm{id} \otimes \Lambda \otimes \mathrm{id})\left(\Delta_{u} \otimes \mathrm{id}\right)(\mathrm{W})=\mathbb{W}_{13} \mathrm{~W}_{23} .
$$

For $\phi \in L^{1}(\mathbb{G})$ and $\sigma \in B\left(L^{2}(\mathbb{G})\right)_{*}$, we then have

$$
(\mathrm{id} \otimes \phi)\left(\Delta_{S}((\mathrm{id} \otimes \sigma) \mathrm{W})\right)=(\mathrm{id} \otimes \sigma)(\mathrm{W}(\phi \otimes \mathrm{id})(\mathrm{W})) .
$$

Since the elements of the form $(\phi \otimes \mathrm{id})(\mathrm{W})$ are dense in $C_{0}(\hat{\mathbb{G}})$ and $C_{0}(\hat{\mathbb{G}})$ is non-degenerate on $L^{2}(\mathbb{G})$, statement (i) follows from the previous equation and the discussion above.

(ii) As $\Delta_{S}(x)$ is in $M\left(C_{0}^{u}(\mathbb{G}) \otimes \mathcal{K}\left(L^{2}(\mathbb{G})\right)\right)$, it follows that $\mathrm{RUC}^{u}(\mathbb{G}) \subseteq M\left(C_{0}^{u}(\mathbb{G})\right)$. That $\operatorname{RUC}^{u}(\mathbb{G})$ is an operator system is obvious.

Remark 1.2. In the case when the multiplicative unitary $\mathrm{W}$ is semi-regular, one can show that $\operatorname{RUC}^{u}(\mathbb{G})$ is in fact a $C^{*}$-algebra: the argument given in $\left[\mathrm{HNR}_{2}\right.$, Theorem 5.6] works also in this case (with some obvious modifications that require, e.g., equation (1.3)).

Similarly, we can define $\operatorname{LUC}^{u}(\mathbb{G})$ starting from the right multiplicative unitary $\mathrm{V} \in M\left(C_{0}\left(\hat{\mathbb{G}}^{\prime}\right) \otimes C_{0}(\mathbb{G})\right)$ and its half-universal lift $\mathrm{V} \in M\left(C_{0}\left(\hat{\mathbb{G}}^{\prime}\right) \otimes C_{0}^{u}(\mathbb{G})\right)$. That is,

$$
\operatorname{LUC}^{u}(\mathbb{G})=\overline{\operatorname{span}}\left\{(\phi \otimes \mathrm{id}) \Delta_{r}(x) \mid \phi \in L^{1}(\mathbb{G}), x \in L^{\infty}(\mathbb{G})\right\},
$$


where $\Delta_{r}(x)=\mathrm{V}(x \otimes 1) \mathrm{V}^{*}$ (note that the right multiplicative unitary $\mathrm{V}$ implements the comultiplication of $\mathbb{G}$ via $\left.\Delta(a)=\mathrm{V}(a \otimes 1) \mathrm{V}^{*}\right)$. The results concerning $\operatorname{RUC}^{u}(\mathbb{G})$ and its dual have obvious analogues for $\operatorname{LUC}^{u}(\mathbb{G})$.

We may view $\mathrm{M}^{u}(\mathbb{G})$ as a subspace of $\mathrm{RUC}^{u}(\mathbb{G})^{*}$, via strict extension.

Every $\rho \in \operatorname{RUC}^{u}(\mathbb{G})^{*}$ defines a map $L_{\rho}: L^{\infty}(\mathbb{G}) \rightarrow L^{\infty}(\mathbb{G})$ by

$$
\phi\left(L_{\rho}(x)\right)=\rho\left((\mathrm{id} \otimes \phi) \Delta_{S}(x)\right) \quad\left(\phi \in L^{1}(\mathbb{G})\right) .
$$

The convolution operators on the universal level are defined in the following proposition.

\section{Proposition 1.3.}

(i) For $\omega \in M^{u}(\mathbb{G})$, the map $R_{\omega}^{u}: M\left(C_{0}^{u}(\mathbb{G})\right) \rightarrow M\left(C_{0}^{u}(\mathbb{G})\right)$,

$$
R_{\omega}^{u}(x)=(\mathrm{id} \otimes \omega) \Delta_{u}(x), \quad x \in M\left(C_{0}^{u}(\mathbb{G})\right),
$$

maps $\mathrm{RUC}^{u}(\mathbb{G})$ to $\mathrm{RUC}^{u}(\mathbb{G})$.

(ii) For $\rho \in \operatorname{RUC}^{u}(\mathbb{G})^{*}$, the equation

$$
\omega\left(L_{\rho}^{u}(x)\right)=\rho\left(R_{\omega}^{u}(x)\right), \quad \omega \in \mathrm{M}^{u}(\mathbb{G}), x \in \mathrm{RUC}^{u}(\mathbb{G})
$$

defines an operator $L_{\rho}^{u}: \mathrm{RUC}^{u}(\mathbb{G}) \rightarrow \mathrm{RUC}^{u}(\mathbb{G})$. Moreover, in the case when $\rho \in \mathrm{M}^{u}(\mathbb{G})$, we have $L_{\rho}^{u}(x)=(\rho \otimes \mathrm{id}) \Delta_{u}(x)$, and $L_{\rho}^{u}$ maps $C_{0}^{u}(\mathbb{G})$ to $C_{0}^{u}(\mathbb{G})$.

Proof. (i) For $\phi \in L^{1}(\mathbb{G})$ and $y \in L^{\infty}(\mathbb{G})$, we have

$$
\begin{aligned}
R_{\omega}^{u}\left((\mathrm{id} \otimes \phi) \Delta_{S}(y)\right) & =(\mathrm{id} \otimes \omega \otimes \phi)\left(\Delta_{u} \otimes \mathrm{id}\right) \Delta_{S}(y) \\
& =(\mathrm{id} \otimes \omega \otimes \phi)\left(\mathrm{id} \otimes \Delta_{S}\right) \Delta_{S}(y) \\
& =(\mathrm{id} \otimes \omega \star \phi) \Delta_{S}(y) .
\end{aligned}
$$

Therefore, $R_{\omega}^{u}$ maps $\operatorname{RUC}^{u}(\mathbb{G})$ to $\mathrm{RUC}^{u}(\mathbb{G})$.

(ii) The equation (1.4) defines an element $L_{\rho}^{u}(x) \in C_{0}^{u}(\mathbb{G})^{* *}$, so it is enough to check that in fact $L_{\rho}^{u}(x) \in \operatorname{RUC}^{u}(\mathbb{G})$. To this end, let $x=(\operatorname{id} \otimes \phi) \Delta_{s}(y)$ for $y \in L^{\infty}(\mathbb{G})$ and $\phi \in L^{1}(\mathbb{G})$. Much as above,

$$
\begin{aligned}
\omega\left(L_{\rho}^{u}(x)\right) & =\rho\left(R_{\omega}^{u}(x)\right)=\rho\left(R_{\omega}^{u}\left((\operatorname{id} \otimes \phi) \Delta_{S}(y)\right)\right)=\rho\left((\mathrm{id} \otimes \omega \star \phi) \Delta_{S}(y)\right) \\
& =\omega \star \phi\left(L_{\rho}(y)\right)=(\omega \otimes \phi) \Delta_{S}\left(L_{\rho}(y)\right)=\omega\left((\operatorname{id} \otimes \phi) \Delta_{S}\left(L_{\rho}(y)\right)\right) .
\end{aligned}
$$

It follows that $L_{\rho}^{u}$ maps $\mathrm{RUC}^{u}(\mathbb{G})$ to itself. The second statement is obvious.

Properties of $\mathrm{RUC}^{u}(\mathbb{G})^{*}$ and the properties of the convolution operators associated with elements in $\mathrm{RUC}^{u}(\mathbb{G})^{*}$ are collected in the following proposition. 


\section{Proposition 1.4.}

(i) $\operatorname{RUC}^{u}(\mathbb{G})^{*}$ is a Banach algebra under the convolution product

$$
\rho \star v=v \circ L_{\rho}^{u}, \quad \rho, v \in \operatorname{RUC}^{u}(\mathbb{G})^{*} .
$$

(ii) $M^{u}(\mathbb{G})$ is a closed subalgebra of $\mathrm{RUC}^{u}(\mathbb{G})^{*}$.

(iii) For every $\omega \in \mathrm{M}^{u}(\mathbb{G})$ and $\rho \in \mathrm{RUC}^{u}(\mathbb{G})^{*}$, we have $\rho \star \omega=\rho \circ R_{\omega}^{u}$.

(iv) For every $\omega, v \in \mathrm{M}^{u}(\mathbb{G})$ and $\rho, \eta \in \mathrm{RUC}^{u}(\mathbb{G})^{*}$,

$$
R_{\omega}^{u} \circ L_{\rho}^{u}=L_{\rho}^{u} \circ R_{\omega}^{u}, \quad R_{\omega}^{u} \circ R_{v}^{u}=R_{\omega \star \nu}^{u}, \quad L_{\eta}^{u} \circ L_{\rho}^{u}=L_{\rho \star \eta}^{u} .
$$

(v) For every $\rho \in \operatorname{RUC}^{u}(\mathbb{G})^{*}$,

$$
\Lambda \circ L_{\rho}^{u}(x)=L_{\rho} \circ \Lambda(x), \quad x \in \operatorname{RUC}^{u}(\mathbb{G}) .
$$

(vi) For every $\rho, \eta \in \mathrm{RUC}^{u}(\mathbb{G})^{*}, L_{\rho \star \eta}=L_{\eta} \circ L_{\rho}$.

(vii) For $\rho \in \operatorname{RUC}^{u}(\mathbb{G})^{*}$, the map $L_{\rho}: L^{\infty}(\mathbb{G}) \rightarrow L^{\infty}(\mathbb{G})$ is normal if and only if $\rho \in \mathrm{M}^{u}(\mathbb{G})$.

(viii) Each of the maps $\omega \mapsto L_{\omega}, \omega \mapsto L_{\omega}^{u}$ and $\omega \mapsto R_{\omega}^{u}$ (the first two defined on $R U C^{u}(\mathbb{G})^{*}$, the last one on $\left.\mathrm{M}^{u}(\mathbb{G})\right)$ are injective.

Proof. (iv) Here, we check the first identity; the second identity is easy to verify, and the third identity is proved in the next paragraph (because it needs the multiplication on $\left.\operatorname{RUC}^{u}(\mathbb{G})^{*}\right)$. For $\omega, v \in \mathrm{M}^{u}(\mathbb{G}), \rho \in \mathrm{RUC}^{u}(\mathbb{G})$ and $x \in \mathrm{RUC}^{u}(\mathbb{G})$, we have

$$
\begin{aligned}
v\left(L_{\rho}^{u} \circ R_{\omega}^{u}(x)\right) & =\rho\left(R_{v}^{u} \circ R_{\omega}^{u}(x)\right)=\rho\left(R_{v \star \omega}^{u}(x)\right) \\
& =v \star \omega\left(L_{\rho}^{u}(x)\right)=v\left(R_{\omega}^{u} \circ L_{\rho}^{u}(x)\right) .
\end{aligned}
$$

(i) It follows from Proposition 1.3 (ii) that $\rho \star \eta=\eta \circ L_{\rho}^{u}$ defines an element of $\operatorname{RUC}^{u}(\mathbb{G})^{*}$ (note that $\mathrm{RUC}^{u}(\mathbb{G}) \subset M\left(C_{0}^{u}(\mathbb{G})\right) \subset C_{0}^{u}(\mathbb{G})^{* *}$ ). Moreover, for every $\rho, \eta, v \in \operatorname{RUC}^{u}(\mathbb{G})$, we have by definition $(\rho \star \eta) \star v=v \circ L_{\rho \star \eta}^{u}$. Now, for every $\omega \in \mathrm{M}^{u}(\mathbb{G})$ and $x \in \mathrm{RUC}^{u}(\mathbb{G})$,

$$
\begin{aligned}
\omega\left(L_{\rho \star \eta}^{u}(x)\right) & =\rho \star \eta\left(R_{\omega}^{u}(x)\right)=\eta\left(L_{\rho}^{u} \circ R_{\omega}^{u}(x)\right) \\
& =\eta\left(R_{\omega}^{u} \circ L_{\rho}^{u}(x)\right)=\omega\left(L_{\eta}^{u} \circ L_{\rho}^{u}(x)\right) .
\end{aligned}
$$

Hence, $L_{\rho \star \eta}^{u}=L_{\eta}^{u} \circ L_{\rho}^{u}$ and

$$
(\rho \star \eta) \star v=\nu \circ L_{\eta}^{u} \circ L_{\rho}^{u}=(\eta \star v) \circ L_{\rho}^{u}=\rho \star(\eta \star v) .
$$

Therefore, the convolution product on $\mathrm{RUC}^{u}(\mathbb{G}) *$ is associative, and clearly the norm is submultiplicative, so $\operatorname{RUC}^{u}(\mathbb{G})^{*}$ is a Banach algebra. 
(ii) is obvious and (iii) is immediate from Proposition 1.3 (ii). Also, (v) is immediate and (vi) is similar to the analogous statement regarding $L_{\rho}^{u}$ in (iv). Condition (viii) is easy to check directly from the definitions.

(vii) If $\rho \in \mathrm{M}^{u}(\mathbb{G})$, then for every $\phi \in L^{1}(\mathbb{G})$ and $x \in L^{\infty}(\mathbb{G})$,

$$
\phi\left(L_{\rho}(x)\right)=\rho\left((\operatorname{id} \otimes \phi) \Delta_{S}(x)\right)=\rho \star \phi(x) .
$$

As $\rho \star \phi \in L^{1}(\mathbb{G})$, it follows that $L_{\rho}$ is normal.

It remains to show that $L_{\rho}$ cannot be normal if $\rho \in C_{0}^{u}(\mathbb{G})^{\perp} \backslash\{0\}$. Now, for every $a \in C_{0}^{u}(\mathbb{G})$, we have $L_{\rho}(\Lambda(a))=\Lambda\left(L_{\rho}^{u}(a)\right)$, and as noted in the proof of Corollary 1.6, $L_{\rho}^{u}(a)=0$. Hence, $L_{\rho}=0$ on $C_{0}(\mathbb{G})$. If $L_{\rho}$ is normal, then $L_{\rho}=0$ because $C_{0}(\mathbb{G})$ is weak ${ }^{*}$-dense in $L^{\infty}(\mathbb{G})$, and consequently $\rho=0$ by (viii).

Sometimes we need to work on $C_{0}^{u}(\mathbb{G})^{* *}$, in which case we may consider normal maps $\widetilde{R_{\omega}^{u}}: C_{0}^{u}(\mathbb{G})^{* *} \rightarrow C_{0}^{u}(\mathbb{G})^{* *}$ and $\widetilde{L_{\omega}^{u}}: C_{0}^{u}(\mathbb{G})^{* *} \rightarrow C_{0}^{u}(\mathbb{G})^{* *}$, given that $\omega \in M^{u}(\mathbb{G})$. These are defined by

$$
\widetilde{R_{\omega}^{u}}(x)=(\mathrm{id} \otimes \omega) \widetilde{\Delta_{u}}(x) \text { and } \widetilde{L_{\omega}^{u}}(x)=(\omega \otimes \mathrm{id}) \widetilde{\Delta_{u}}(x) .
$$

Note that on $M\left(C_{0}^{u}(\mathbb{G})\right)$ the maps $\widetilde{R_{\omega}^{u}}$ and $\widetilde{L_{\omega}^{u}}$ agree with the maps $R_{\omega}^{u}$ and $L_{\omega}^{u}$, respectively.

We will need one more result, essentially contained in Proposition 2.1 of [BMS]; here we provide a different proof.

Proposition 1.5. Let $A$ be a $C^{*}$-algebra. Then, $M(A)^{*}=A^{*} \oplus_{1} A^{\perp}$, where as usual $A^{*} \subset M(A)^{*}$ is defined using strict extensions of functionals in $A^{*}$.

Proof. For every $v \in A^{*}$, denote its strict extension in $M(A)^{*}$ by $\tilde{v}$. For every $\mu \in M(A)^{*}$ define $\mu_{0}=\left.\mu\right|_{A}$ and $\mu_{1}=\mu-\tilde{\mu}_{0}$. Then, $\mu=\tilde{\mu}_{0}+\mu_{1}$ will be the required decomposition.

Suppose first that $\mu$ is a positive functional. Then, clearly also $\tilde{\mu}_{0}$ is positive. We claim that $\tilde{\mu}_{0} \leq \mu$ so that also $\mu_{1}$ is positive. Let $x \geq 0$ in $M(A)$. If $\left(e_{i}\right)_{i \in \mathcal{I}}$ is an increasing, positive contractive approximate identity in $A$, then $\left(x^{1 / 2} e_{i} x^{1 / 2}\right)_{i \in \mathcal{I}}$ is an increasing net converging strictly to $x$. Hence,

$$
\tilde{\mu}_{0}(x)=\lim _{i \in \mathcal{I}} \mu_{0}\left(x^{1 / 2} e_{i} x^{1 / 2}\right)=\lim _{i \in \mathcal{I}} \mu\left(x^{1 / 2} e_{i} x^{1 / 2}\right) \leq \mu(x) .
$$

Thus, $\mu=\tilde{\mu}_{0}+\mu_{1}$ is a decomposition of $\mu$ into positive components, and so

$$
\|\mu\|=\mu(1)=\tilde{\mu}_{0}(1)+\mu_{1}(1)=\left\|\tilde{\mu}_{0}\right\|+\left\|\mu_{1}\right\| .
$$

Therefore, the claim holds for positive $\mu$.

Let now $\mu \in M(A)^{*}$ be arbitrary. Then, $\mu$ has a (right) polar decomposition $\mu=\nu . u$ where $\nu \in M(A)^{*}$ is positive and $u \in M(A)^{* *}$ is a partial isometry 
(the polar decomposition follows by considering $M(A)^{*}$ as the predual of the von Neumann algebra $\left.M(A)^{* *}\right)$.

We claim that $\tilde{v}_{0} \cdot u=\tilde{\mu}_{0}$. These functionals coincide on $A$, so it is enough to show that $\bar{v}_{0} . u$ is strictly continuous. As there are two different extensions at play (from $A$ to $M(A)$ and from $M(A)$ to $M(A)^{* *}$ ), it is clearer to use angle brackets to denote the duality of $M(A)^{*}$ and $M(A)^{* *}$. Let $v_{0}=a . \eta$ be a factorisation of $v_{0}$ on $A\left(\eta \in A^{*}\right.$ and $\left.a \in A\right)$. The functional $\tilde{v}_{0}$ is defined by

$$
\tilde{v}_{0}(y)=\eta(y a), \quad y \in M(A),
$$

so that by a weak*-approximation we have also $\tilde{v}_{0}(z)=\tilde{\eta}(z a)$ for all $z \in$ $M(A)^{* *}$, where $\tilde{\eta}$ denotes the relevant weak*-extension. Let $\left(x_{i}\right)_{i \in \mathcal{I}} \subset M(A)$ converge strictly to $x$ in $M(A)$. Now, we have

$$
\left\langle\tilde{v}_{0}, u x\right\rangle=\langle\tilde{\eta}, u x a\rangle=\left\langle\tilde{\eta}, u\left(\lim _{i \in \mathcal{I}} x_{i} a\right)\right\rangle=\lim _{i \in \mathcal{I}}\left\langle\tilde{\eta}, u x_{i} a\right\rangle=\lim _{i \in \mathcal{I}}\left\langle\tilde{v}_{0}, u x_{i}\right\rangle,
$$

which shows that $\tilde{v}_{0} . u$ is strictly continuous, as required.

As $\tilde{v}_{0} \cdot u=\tilde{\mu}_{0}$, it follows from the uniqueness of the decomposition that $v_{1} \cdot u=\mu_{1}$. Then, we have

$$
\|\mu\| \leq\left\|\tilde{\mu}_{0}\right\|+\left\|\mu_{1}\right\|=\left\|\tilde{v}_{0} \cdot u\right\|+\left\|v_{1} \cdot u\right\| \leq\left\|\tilde{v}_{0}\right\|+\left\|v_{1}\right\|=\|v\|=\|\mu\|,
$$

where we used the first part of the proof (applied to the positive functional $v$ ) and the fact that $\|\mu\|=\|v\|$.

Corollary 1.6. We have a decomposition $\operatorname{RUC}^{u}(\mathbb{G})^{*}=M^{u}(\mathbb{G}) \oplus_{1} C_{0}^{u}(\mathbb{G})^{\perp}$ where $M^{u}(\mathbb{G})$ is a subalgebra of $\operatorname{RUC}^{u}(\mathbb{G})^{*}$ and $C_{0}^{u}(\mathbb{G})^{\perp}$ is a weak $k^{*}$-closed ideal in $\operatorname{RUC}^{u}(\mathbb{G})^{*}$.

Proof. It remains still to show that $C_{0}^{u}(\mathbb{G})^{\perp}$ is an ideal. Suppose first that $\mu, \nu \in \mathrm{RUC}^{u}(\mathbb{G})^{*}$ with $\mu \in C_{0}^{u}(\mathbb{G})^{\perp}$. For all $\omega \in \mathrm{RUC}^{u}(\mathbb{G})^{*}$ and $a \in C_{0}^{u}(\mathbb{G})$, we have $\omega\left(L_{\mu}^{u}(a)\right)=\mu\left(R_{\omega}^{u}(a)\right)=0$ as $R_{\omega}^{u}(a) \in C_{0}^{u}(\mathbb{G})$, and so $L_{\mu}^{u}(a)=0$. It follows that $\mu \star v=v \circ L_{\mu}^{u} \in C_{0}^{u}(\mathbb{G})^{\perp}$.

It remains to check the case when $\mu \in \operatorname{RUC}^{u}(\mathbb{G})^{*}$ and $\nu \in C_{0}^{u}(\mathbb{G})^{\perp}$. But now $L_{\mu}^{u}(a) \in C_{0}^{u}(\mathbb{G})$ whenever $a \in C_{0}^{u}(\mathbb{G})$, and so $\mu \star v \in C_{0}^{u}(\mathbb{G})^{\perp}$.

Lemma 1.7. If $\omega \in \mathrm{RUC}^{u}(\mathbb{G})^{*}$ is a contractive idempotent, then we have either $\omega \in \mathrm{M}^{u}(\mathbb{G})$ or $\omega \in C_{0}^{u}(\mathbb{G})^{\perp}$.

Proof. Write $\omega=\omega_{0}+\omega_{1}$ where $\omega_{0} \in \mathrm{M}^{u}(\mathbb{G})$ and $\omega_{1} \in C_{0}^{u}(\mathbb{G})^{\perp}$. Then,

$$
\omega_{0}+\omega_{1}=\omega=\omega \star \omega=\omega_{0} \star \omega_{0}+\omega_{0} \star \omega_{1}+\omega_{1} \star \omega_{0}+\omega_{1} \star \omega_{1} .
$$

Since $C_{0}(\mathbb{G})^{\perp}$ is an ideal, it follows that $\omega_{0} \star \omega_{0}=\omega_{0}$. Now $\|\omega\|=\left\|\omega_{0}\right\|+\left\|\omega_{1}\right\|$ by Corollary 1.6, so $\omega_{0}$ is a contractive idempotent. Hence, $\left\|\omega_{0}\right\|$ is either 0 or 1. If $\left\|\omega_{0}\right\|=0$, then $\omega=\omega_{1} \in C_{0}^{u}(\mathbb{G})^{\perp}$. If $\left\|\omega_{0}\right\|=1$, then $\left\|\omega_{1}\right\|=0$ and $\omega=\omega_{0} \in M^{u}(\mathbb{G})$. 


\section{FIXED POINTS IN $L^{\infty}(\mathbb{G})$}

Let $\mathbb{G}$ be a locally compact quantum group. For a map $L: L^{\infty}(\mathbb{G}) \rightarrow L^{\infty}(\mathbb{G})$, write

$$
\text { Fix } L=\left\{x \in L^{\infty}(\mathbb{G}) \mid L(x)=x\right\} .
$$

We are mostly interested in Fix $L_{\omega}$ with $\omega \in \mathrm{M}^{u}(\mathbb{G})_{1}$, in which case Fix $L_{\omega}$ is weak ${ }^{*}$-closed. Note that if $\|\omega\|<1$, then Fix $L_{\omega}=\{0\}$, so we may concentrate on the case $\|\omega\|=1$.

Given $\omega \in \mathrm{M}^{u}(\mathbb{G})_{1}$ and $n \in \mathbb{N}$ we write

$$
S_{n}(\omega)=\frac{1}{n} \sum_{k=1}^{n} \omega^{\star k} \in \mathrm{M}^{u}(\mathbb{G})_{1} .
$$

Fix a free ultrafilter $p$ on $\mathbb{N}$ and take the weak* limit of the sequence $S_{n}(\omega)$ along $p$ in $\operatorname{RUC}^{u}(\mathbb{G})^{*}$. We will denote this limit by $\tilde{\omega}_{p}$ (later we will simply write $\tilde{\omega}$ once $p$ is fixed).

Lemma 2.1. Let $\omega \in \mathrm{M}^{u}(\mathbb{G})_{1}$ and let $p$ be a free ultrafilter. Then,

$$
\tilde{\omega}_{p}=\omega \star \tilde{\omega}_{p}=\tilde{\omega}_{p} \star \omega=\tilde{\omega}_{p} \star \tilde{\omega}_{p} .
$$

In particular, $\tilde{\omega}_{p}$ is either 0 or a contractive idempotent. Finally, the following conditions are also equivalent:

(i) $\tilde{\omega}_{p}$ is a state for some free ultrafilter $p$.

(ii) $\tilde{\omega}_{p}$ is a state for every free ultrafilter $p$.

(iii) $\omega$ is a state.

Proof. The first statement follows from the identity

$$
S_{n}(\omega) \star \omega=\omega \star S_{n}(\omega)=S_{n}(\omega)-\frac{1}{n} \omega+\frac{1}{n} \omega^{n+1} .
$$
Then,

The implications (iii) $\Longrightarrow$ (ii) $\Longrightarrow$ (i) are obvious. Suppose that $\tilde{\omega}_{p}$ is a state.

$$
1=\tilde{\omega}_{p}(1)=\omega \star \tilde{\omega}_{p}(1)=\omega(1) \tilde{\omega}_{p}(1)=\omega(1),
$$

so (iii) follows.

Lemma 2.2. Let $\omega \in \mathrm{M}^{u}(\mathbb{G})_{1}$ and let $p$ be a free ultrafilter. The map $L_{\bar{\omega}_{p}}$ is a projection onto Fix $L_{\omega}=\operatorname{Fix} L_{\tilde{\omega}_{p}}$.

Proof. To see that $L_{\bar{\omega}_{p}}$ and $L_{\omega}$ have the same fixed points, note first that for all $\phi \in L^{1}(\mathbb{G})$ and $x \in L^{\infty}(\mathbb{G})$,

$$
\begin{aligned}
\phi\left(L_{\tilde{\omega}_{p}}(x)\right) & =\tilde{\omega}_{p}\left((\operatorname{id} \otimes \phi) \Delta_{S}(x)\right) \\
& =p-\lim S_{n}(\omega)\left((\operatorname{id} \otimes \phi) \Delta_{S}(x)\right) .
\end{aligned}
$$


It is immediate that Fix $L_{\omega} \subset$ Fix $L_{\tilde{\omega}_{p}}$. On the other hand if $x$ is a fixed point of $L_{\tilde{\omega}_{p}}$, then it follows from the above identity that, by $(2.1)$,

$$
\begin{aligned}
\phi\left(L_{\omega}(x)\right) & =\phi\left(L_{\omega}\left(L_{\tilde{\omega}_{p}}(x)\right)\right)=\phi\left(L_{\tilde{\omega}_{p} \star \omega}(x)\right) \\
& =\tilde{\omega}_{p} \star \omega\left((\operatorname{id} \otimes \phi) \Delta_{S}(x)\right) \\
& =p-\lim S_{n}(\omega)\left((\operatorname{id} \otimes(\omega \star \phi)) \Delta_{S}(x)\right) \\
& =p-\lim \left(S_{n}(\omega) \star \omega\right)\left((\operatorname{id} \otimes \phi) \Delta_{s}(x)\right) \\
& =\phi\left(L_{\tilde{\omega}_{p}}(x)\right)=\phi(x) .
\end{aligned}
$$

Since $\tilde{\omega}_{p}$ is either a non-zero contractive idempotent or 0 , it follows that $L_{\tilde{\omega}_{p}}$ is an idempotent whose image is Fix $L_{\tilde{\omega}_{p}}$.

The following theorem characterises the case when there are no non-zero fixed points. Note that convolution operators determined by states always have fixed points (namely, the constants), but this need not be the case with contractive functionals.

Theorem 2.3. Let $\omega \in \mathrm{M}^{u}(\mathbb{G})_{1}$. The following are equivalent:

(i) $L_{\omega}$ has no non-zero fixed points in $L^{\infty}(\mathbb{G})$.

(ii) $L_{\omega}$ has no non-zero fixed points in $\operatorname{RUC}(\mathbb{G})$.

(iii) $L_{\omega}^{u}$ has no non-zero fixed points in $\mathrm{RUC}^{u}(\mathbb{G})$.

(iv) $S_{n}(\omega) \rightarrow 0$ weak* in $\mathrm{RUC}^{u}(\mathbb{G})^{*}$.

(v) $\tilde{\omega}_{p}=0$ for all free ultrafilters $p$.

(vi) $\tilde{\omega}_{p}=0$ for some free ultrafilter $p$.

Proof. (iii) $\Longrightarrow$ (i), as follows. Let $x$ be a non-zero fixed point of $L_{\omega}$ in $L^{\infty}(\mathbb{G})$, and pick $\phi \in L^{1}(\mathbb{G})$ such that

$$
\tilde{x}:=(\mathrm{id} \otimes \phi) \Delta_{S}(x) \neq 0 .
$$

Then, $\tilde{x} \in \operatorname{RUC}^{u}(\mathbb{G})$, and for every $\mu \in \operatorname{RUC}^{u}(\mathbb{G})^{*}$,

$$
\begin{aligned}
\mu\left(L_{\omega}^{u}(\tilde{x})\right) & =\omega \star \mu\left((\operatorname{id} \otimes \phi) \Delta_{S}(x)\right)=\phi\left(L_{\omega \star \mu}(x)\right) \\
& =\phi\left(L_{\mu} \circ L_{\omega}(x)\right)=\phi\left(L_{\mu}(x)\right)=\mu(\tilde{x}) .
\end{aligned}
$$

Thus, $\tilde{x}$ is a non-zero fixed point of $L_{\omega}^{u}$ in $\operatorname{RUC}^{u}(\mathbb{G})$.

(ii) $\Longrightarrow$ (i) is similar to (iii) $\Longrightarrow$ (i). The converse implication (i) $\Longrightarrow$ (ii) is trivial. The implications (iv) $\Longleftrightarrow$ (v) $\Longrightarrow$ (vi) are obvious.

(i) $\Longrightarrow$ (v): If $\tilde{\omega}_{p} \neq 0$, there is $x \in L^{\infty}(\mathbb{G})$ such that $L_{\tilde{\omega}_{p}}(x) \neq 0$. Moreover, $L_{\omega}\left(L_{\bar{\omega}_{p}}(x)\right)=L_{\tilde{\omega}_{p}}(x)$.

(vi) $\Longrightarrow$ (iii), as follows. Suppose that there exists a non-zero fixed point $x$ of $L_{\omega}^{u}$ in $\operatorname{RUC}^{u}(\mathbb{G})$, and pick $v \in \mathrm{M}^{u}(\mathbb{G})$ such that we have $v(x) \neq 0$ (recall 
that $\left.\operatorname{RUC}^{u}(\mathbb{G}) \subset M\left(C_{0}^{u}(\mathbb{G})\right) \subset C_{0}^{u}(\mathbb{G})^{* *}\right)$. Then, using Proposition 1.4, we have

$$
\begin{aligned}
v\left(L_{\tilde{\omega}_{p}}^{u}(x)\right) & =\tilde{\omega}_{p} \star v(x)=\tilde{\omega}_{p}\left(R_{v}^{u}(x)\right)=p-\lim S_{n}(\omega)\left(R_{v}^{u}(x)\right) \\
& =p-\lim \frac{1}{n} \sum_{k=1}^{n} v\left(L_{\omega^{\star k}}^{u}(x)\right)=v(x) .
\end{aligned}
$$

It follows that $\tilde{\omega}_{p} \neq 0$.

The right absolute value of $\omega \in \mathrm{M}^{u}(\mathbb{G})$ is a positive functional $|\omega|$ in $M^{u}(\mathbb{G})$ defined through the polar decomposition $\omega=|\omega|(\cdot u)$ where $u \in C_{0}^{u}(\mathbb{G})^{* *}$ is a partial isometry satisfying some extra properties (see Definition III.4.3 of [Tak]). Then, the left absolute value $|\omega|_{\ell}$ is defined by $\omega=|\omega|_{\ell}\left(u^{*} \cdot\right)$.

Lemma 2.4. Let $M$ be a von Neumann algebra and $v \in M_{*}$ with $\|v\|=1$. Suppose $x \in M$ is such that $\|x\|=1$ and $v\left(x^{*}\right)=1$. Then, $v=x \cdot|v|$ and $|v|=x^{*} . v$.

Proof. By the proof of Theorem III.4.2 of [Tak], $v=u .|v|$ where $x=u|x|$ is the polar decomposition of $x$. Note that $|v|(|x|)=v\left(|x| u^{*}\right)=1$. By the Cauchy-Schwarz inequality,

$$
|v|(|x|)^{2} \leq|v|\left(|x|^{2}\right)|v|(1) \leq 1=|v|(|x|)^{2} .
$$

Hence, $|x|$ is in the multiplicative domain of $|v|$, and so for every $y \in M$,

$$
x .|v|(y)=|v|(y u|x|)=|v|(y u)|v|(|x|)=v(y) .
$$

As $|v|=u^{*} . v$, we also have $x^{*} . v(y)=v\left(y|x| u^{*}\right)=|v|(y|x|)=|v|(y)$.

The above general lemma allows us to say something about fixed points of contractive convolution operators.

Lemma 2.5. Suppose that $\omega \in \mathrm{M}^{u}(\mathbb{G})$ is contractive and $v \in C_{0}^{u}(\mathbb{G})^{* *}$ is such that $v^{*}$ is a fixed point of $\widetilde{L_{\omega}^{u}},\|v\|=1$ and $\omega\left(v^{*}\right)=1$. Then,

$$
{\widetilde{\Delta_{u}}}^{(k)}(v)-v^{\otimes(k+1)} \in N_{|\omega|^{\otimes(k+1)}} \quad \text { and } \quad \omega^{\star k}=v \cdot|\omega|^{\star k}
$$

for every $k \in \mathbb{N}$. Moreover, $|\omega|^{\star k}\left(a v^{*} v\right)=|\omega|^{\star k}\left(v^{*} v a\right)=|\omega|^{\star k}(a)$ for every $a \in C_{0}^{u}(\mathbb{G})^{* *}$ and $k \in \mathbb{N}$.

Proof. As $\|v\|=1$ and $\omega\left(v^{*}\right)=1$, it follows from Lemma 2.4 that $\omega=$ $v .|\omega|$. Moreover, $|\omega|\left(v^{*} v\right)=1$.

Fix $k \in \mathbb{N}$. Since $v^{*}$ is a fixed point of $\widetilde{L_{\omega}^{u}}$, we have

$$
1=\omega\left(v^{*}\right)=\omega^{\otimes(k+1)}\left({\widetilde{\Delta_{u}}}^{(k)}\left(v^{*}\right)\right)=|\omega|^{\otimes(k+1)}\left({\widetilde{\Delta_{u}}}^{(k)}\left(v^{*}\right) v^{\otimes(k+1)}\right) .
$$


Therefore,

$$
\begin{aligned}
& |\omega|^{\otimes(k+1)}\left(\left({\widetilde{\Delta_{u}}}^{(k)}(v)-v^{\otimes(k+1)}\right)^{*}\left({\widetilde{\Delta_{u}}}^{(k)}(v)-v^{\otimes(k+1)}\right)\right) \\
& =|\omega|^{\otimes(k+1)}{\widetilde{\widetilde{\Delta}_{u}}}^{(k)}\left(v^{*} v\right)-{\widetilde{\Delta_{u}}}^{(k)}\left(v^{*}\right) v^{\otimes(k+1)} \\
& \left.\quad-\left(v^{*}\right)^{\otimes(k+1)}{\widetilde{\Delta_{u}}}^{(k)}(v)+\left(v^{*} v\right)^{\otimes(k+1)}\right) \\
& \left.=|\omega|^{\otimes(k+1)}{\widetilde{\Delta_{u}}}^{(k)}\left(v^{*} v\right)\right)-1-1+1=0 .
\end{aligned}
$$

Thus, we have

$$
{\widetilde{\Delta_{u}}}^{(k)}(v)-v^{\otimes(k+1)} \in N_{|\omega| \otimes(k+1)},
$$

and it is then immediate that $\omega^{\star k}=v \cdot|\omega|^{\star k}$.

As a byproduct, we get that $|\omega|^{\star k}\left(v^{*} v\right)=1$, and so by the Cauchy-Schwarz inequality,

$$
1=|\omega|^{\star k}\left(v^{*} v\right)^{2} \leq|\omega|^{\star k}\left(v^{*} v v^{*} v\right) \leq\left\|v^{*} v v^{*} v\right\| \leq 1 .
$$

By Choi's theorem on multiplicative domains, $v^{*} v$ is in the multiplicative domain of $|\omega|^{\star k}$, and so $|\omega|^{\star k}\left(a v^{*} v\right)=|\omega|^{\star k}\left(v^{*} v a\right)=|\omega|^{\star k}(a)$.

In the case when $\omega$ is a state, the next lemma is trivial (one can take $v=1$ ).

Lemma 2.6. Let $\omega \in \mathrm{M}^{u}(\mathbb{G})_{1}$. If $L_{\omega}$ has a non-zero fixed point in $L^{\infty}(\mathbb{G})$, then there exists $v \in C_{0}^{u}(\mathbb{G})^{* *}$ such that

$$
{\widetilde{\Delta_{u}}}^{(k)}(v)-v^{\otimes k+1} \in N_{|\omega|^{\otimes k+1}} \quad \text { and } \quad \omega^{\star k}=v \cdot|\omega|^{\star k}
$$

for every $k \in \mathbb{N}$. Moreover, $|\omega|^{\star k}\left(a v^{*} v\right)=|\omega|^{\star k}\left(v^{*} v a\right)=|\omega|^{\star k}(a)$ for every $a \in C_{0}^{u}(\mathbb{G})$ and $k \in \mathbb{N}$.

Proof. Let $x \in L^{\infty}(\mathbb{G})$ be a non-zero fixed point of $L_{\omega}$. Pick a sequence $\left(\phi_{n}\right)_{n \in \mathbb{N}} \subseteq$ ball $\left(L^{1}(\mathbb{G})\right)$ such that $\phi_{n}(x)>\|x\|-1 / n$ for each $n \in \mathbb{N}$. Define

$$
v_{n}=\frac{\left(\mathrm{id} \otimes \phi_{n}^{*}\right) \Delta_{s}\left(x^{*}\right)}{\phi_{n}^{*}\left(x^{*}\right)} \in \mathrm{RUC}^{u}(\mathbb{G}),
$$

and let $v$ be a weak* cluster point of $\left(v_{n}\right)$ in $C_{0}^{u}(\mathbb{G})^{* *}$. Since $L_{\omega}(x)=x$, it follows that $\omega\left(v_{n}^{*}\right)=1$ for every $n \in \mathbb{N}$, and so $\omega\left(v^{*}\right)=1$. Moreover, since $\phi_{n}(x) \rightarrow\|x\|$, it follows that $\|v\| \leq 1$, and so $\|v\|=1$. Finally, we note that $v^{*}$ is a fixed point of $\widetilde{L_{\omega}^{u}}$ : indeed, it is easy to calculate that $L_{\omega}^{u}\left(v_{n}^{*}\right)=v_{n}^{*}$, and the rest follows as $\widetilde{L_{\omega}^{u}}$ is normal when $\omega \in \mathrm{M}^{u}(\mathbb{G})$. Now, we may apply Lemma 2.5 to obtain the result. 
In the case when $L_{\omega}^{u}$ has a non-zero fixed point in $\operatorname{LUC}^{u}(\mathbb{G})$, we obtain a tighter result. Note that by Theorem 2.3 the existence of a non-zero fixed point in $L^{\infty}(\mathbb{G})$ implies the existence of a non-zero fixed point in $\operatorname{RUC}^{u}(\mathbb{G})$ but not necessarily in $\operatorname{LUC}^{u}(\mathbb{G})$.

We call a contractive functional $\omega \in \mathrm{M}^{u}(\mathbb{G})$ non-degenerate if both $|\omega|$ and $|\omega|_{\ell}$ are non-degenerate in the sense of $\left[\mathrm{KNR}_{1}\right]$; that is, for each non-zero $x \in C_{0}^{u}(\mathbb{G})_{+}$there exists $n \in \mathbb{N}$ such that $|\omega|^{\star n}(x)>0$ and $|\omega|_{\ell}^{\star n}(x)>0$. We use the analogous notion of degeneracy for $\omega \in \mathrm{M}(\mathbb{G})$.

Theorem 2.7. Suppose that $\omega \in \mathrm{M}^{u}(\mathbb{G})$ is non-degenerate and contractive. If $L_{\omega}^{u}$ has a non-zero fixed point in $\operatorname{LUC}^{u}(\mathbb{G})$, then there is a unitary $v \in \operatorname{LUC}^{u}(\mathbb{G})$ $\left(\right.$ even $\left.\mathrm{UC}^{u}(\mathbb{G})\right)$ such that $\Delta_{u}(v)=v \otimes v$ and $\operatorname{Fix} L_{\omega}^{u}=\left(\operatorname{Fix} L_{|\omega|}^{u}\right) v^{*}$. Taking $u=\Lambda(v)$, we have Fix $L_{\omega}=\left(\operatorname{Fix} L_{|\omega|}\right) u^{*}$.

Proof. Let $x \in \operatorname{LUC}^{u}(\mathbb{G})$ be a fixed point of $L_{\omega}^{u}$ such that $\|x\|=1$. We fix $\mu \in \operatorname{LUC}^{u}(\mathbb{G})^{*}$ so that $\|\mu\|=1$ and $\mu(x)=1$. Define $v=R_{\mu}^{u}(x)^{*} \in \operatorname{LUC}^{u}(\mathbb{G})$ (where $R_{\mu}^{u}$ is defined analogously to $L_{\mu}^{u}$, introduced in Proposition 1.4). Then, $\omega\left(v^{*}\right)=\omega\left(R_{\mu}^{u}(x)\right)=\mu\left(L_{\omega}^{u}(x)\right)=\mu(x)=1$, and in particular $\|v\|=1$. Moreover, $v^{*}$ is a fixed point of $L_{\omega}^{u}$ as $L_{\omega}^{u}$ commutes with $R_{\mu}^{u}$. Now we may apply Lemma 2.5.

We claim that $\Delta_{u}(v)=v \otimes v$. For every $k, l \in \mathbb{N}$, we have

$$
\begin{gathered}
|\omega|^{\star k} \otimes|\omega|^{\star l}\left(\left(\Delta_{u}(v)-v \otimes v\right)^{*}\left(\Delta_{u}(v)-v \otimes v\right)\right) \\
=|\omega|^{\star k} \otimes|\omega|^{\star l}\left(\Delta_{u}\left(v^{*} v\right)-\Delta_{u}\left(v^{*}\right)(v \otimes v)\right. \\
\left.-\left(v^{*} \otimes v^{*}\right) \Delta_{u}(v)+v^{*} v \otimes v^{*} v\right) \\
=|\omega|^{\star(k+l)}\left(v^{*} v\right)-\omega^{\star(k+l)}\left(v^{*}\right)-\overline{\omega^{\star(k+l)}\left(v^{*}\right)} \\
+|\omega|^{\star k}\left(v^{*} v\right)|\omega|^{\star l}\left(v^{*} v\right)=0 .
\end{gathered}
$$

Now, $X=\left(\Delta_{u}(v)-v \otimes v\right)^{*}\left(\Delta_{u}(v)-v \otimes v\right) \in M\left(C_{0}^{u}(\mathbb{G}) \otimes C_{0}^{u}(\mathbb{G})\right)$ is such that $X \geq 0$ and $|\omega|^{\star k} \otimes|\omega|^{\star l}(X)=0$. Since for every $l \in \mathbb{N}$

$$
|\omega|^{\star l}\left(\left(|\omega|^{\star k} \otimes \mathrm{id}\right)(X)\right)=0,
$$

it follows by non-degeneracy that $\left(|\omega|^{\star k} \otimes\right.$ id $)(X)=0$. Then, for every $\sigma \geq 0$ in $C_{0}^{u}(\mathbb{G})^{*}$, we have $(\operatorname{id} \otimes \sigma)(X) \geq 0$ and $|\omega|^{\star k}((\operatorname{id} \otimes \sigma)(X))=0$ for every $k \in \mathbb{N}$. Therefore, $(\mathrm{id} \otimes \sigma)(X)=0$ for every $\sigma \geq 0$, and so $X=0$. Consequently, $\Delta_{u}(v)=v \otimes v$ as required.

Now, for every $a \in C_{0}^{u}(\mathbb{G})$ we have

$$
\begin{aligned}
& |\omega|^{\star k}\left(\left(a v^{*} v-a\right)^{*}\left(a v^{*} v-a\right)\right) \\
& \quad=|\omega|^{\star k}\left(v^{*} v a^{*} a v^{*} v-v^{*} v a^{*} a-a^{*} a v^{*} v+a^{*} a\right)=0 .
\end{aligned}
$$


Non-degeneracy implies that $a v^{*} v=a$, and then it follows that $v^{*} v=1$. Similarly, $v v^{*}=1$ follows by applying non-degeneracy of the lefthand-sided absolute value $|\omega|_{\ell}$.

If $y \in$ Fix $L_{|\omega|}^{u}$, then

$$
\begin{aligned}
L_{\omega}^{u}\left(y v^{*}\right) & =(\omega \otimes \mathrm{id}) \Delta_{u}\left(y v^{*}\right) \\
& =(\omega \otimes \mathrm{id})\left(\Delta_{u}(y)\left(v^{*} \otimes v^{*}\right)\right) \\
& =(|\omega| \otimes \mathrm{id})\left(\Delta_{u}(y)\right) v^{*}=y v^{*} .
\end{aligned}
$$

Hence, Fix $L_{|\omega|}^{u} v^{*} \subset$ Fix $L_{\omega}^{u}$. Conversely, if $z \in \operatorname{Fix} L_{\omega}^{u}$, then

$$
\begin{aligned}
L_{|\omega|}^{u}(z v) & =(|\omega| \otimes \mathrm{id}) \Delta_{u}(z v) \\
& =(|\omega| \otimes \mathrm{id})\left(\Delta_{u}(z)(v \otimes v)\right) \\
& =(\omega \otimes \mathrm{id})\left(\Delta_{u}(z)\right) v=z v .
\end{aligned}
$$

Therefore, Fix $L_{\omega}^{u}=\left(\operatorname{Fix} L_{|\omega|}^{u}\right) v^{*}$ and Fix $L_{|\omega|}^{u}=\left(\operatorname{Fix} L_{\omega}^{u}\right) v$.

Finally, $u=\Lambda(v)$ is a group-like unitary in $M\left(C_{0}(\mathbb{G})\right)$ and $u^{*}$ is a fixed point of $L_{\omega}$. Much as above, Fix $L_{\omega}=\left(\operatorname{Fix} L_{|\omega|}\right) u^{*}$ and Fix $L_{|\omega|}=\left(\operatorname{Fix} L_{\omega}\right) u$.

We say that a locally compact quantum group $\mathbb{G}$ is universally SIN if we have that $\operatorname{LUC}^{u}(\mathbb{G})=\mathrm{RUC}^{u}(\mathbb{G})$. Note that all discrete and compact quantum groups are universally SIN, as are duals of locally compact groups. Recall that $\mathrm{Hu}$, Neufang, and Ruan called a locally compact quantum group SIN (from "having small invariant neighbourhoods") if LUC( $\mathbb{G})=\operatorname{RUC}(\mathbb{G})$. If $\mathbb{G}$ is universally SIN, it is SIN, and the two notions trivially coincide for coamenable $\mathbb{G}$. We do not know if they coincide in general.

A weak*-closed subspace of a von Neumann algebra is called a $W^{*}$-sub-TRO if it is closed under the ternary product $(x, y, z) \mapsto x y^{*} z$.

Corollary 2.8. Suppose that $\mathbb{G}$ is a locally compact quantum group that is universally $S I N$, and let $\omega \in \mathrm{M}^{u}(\mathbb{G})_{1}$ be non-degenerate. If Fix $L_{\omega}$ is a $W^{*}$-sub-TRO of $L^{\infty}(\mathbb{G})$, then $\operatorname{dim}\left(\operatorname{Fix} L_{\omega}\right) \leq 1$ (moreover, if $\operatorname{Fix} L_{\omega}$ is non-zero, it contains a unitary).

Proof. The case when Fix $L_{\omega}=\{0\}$ is clear, so we may suppose Fix $L_{\omega} \neq\{0\}$. We then first use Theorem 2.3 and the assumption that $\mathbb{G}$ is universally SIN to deduce that the assumptions of Theorem 2.7 hold. Thus, there is a group-like unitary $u$ such that Fix $L_{\omega}=\left(\operatorname{Fix} L_{|\omega|}\right) u^{*}$. If Fix $L_{\omega}$ is a $W^{*}$-sub-TRO, then Fix $L_{|\omega|}$ is a von Neumann subalgebra. Hence, Fix $L_{|\omega|} \cong \mathbb{C}$ by Theorem 3.6 in $\left[\mathrm{KNR}_{1}\right]$.

We do not know if the universally SIN assumption of the previous result is actually necessary; our methods seem, however, to be limited to this case. 


\section{ANNIHILATORS OF Fix $L_{\omega}$ IN $L^{1}(\mathbb{G})$}

In this short section, we describe properties of the pre-annihilators of fixed point spaces of the type Fix $L_{\omega} \subseteq L^{\infty}(\mathbb{G})$, that is, certain one-sided ideals in $L^{1}(\mathbb{G})$.

Definition 3.1. Given $\omega \in \mathrm{P}^{u}(\mathbb{G})$, define a closed right ideal $I_{\omega}$ in $L^{1}(\mathbb{G})$ by the formula

$$
I_{\omega}:=\operatorname{cl}\left\{f-\omega \star f \mid f \in L^{1}(\mathbb{G})\right\} .
$$

Moreover, let $L_{0}^{1}(\mathbb{G})$ denote the augmentation ideal in $L^{1}(\mathbb{G})$, that is, $L_{0}^{1}(\mathbb{G})=$ $\left\{f \in L^{1}(\mathbb{G}) \mid f(1)=0\right\}$.

Lemma 3.2. Let $\omega \in \mathrm{P}^{u}(\mathbb{G})$. Then, the right ideal $I_{\omega}$ is equal to the preannibilator of Fix $L_{\omega}$. In particular, $I_{\omega} \subset L_{0}^{1}(\mathbb{G})$.

Proof. To show the first statement, it suffices to consider the following chain of equivalences:

$$
\begin{aligned}
x \in I_{\omega}^{\perp} & \Longleftrightarrow \forall_{f \in L^{1}(\mathbb{G})} f(x)-\omega \star f(x)=0 \\
& \Longleftrightarrow \forall_{f \in L^{1}(\mathbb{G})} f(x)=f\left(L_{\omega}(x)\right) \Longleftrightarrow x=L_{\omega}(x) .
\end{aligned}
$$

The second statement is now obvious.

The following result can be proved exactly as its classical counterpart, that is, [Wil, Theorem 1.2]. We outline the argument for completeness.

Proposition 3.3. Assume that $\mathbb{G}$ is second countable (i.e., $C_{0}(\mathbb{G})$ is separable), and let $\mathcal{I}=\left\{I_{\omega} \mid \omega \in \mathrm{P}^{u}(\mathbb{G})\right\}$. Then, each element in $\mathcal{I}$ is contained in a maximal element of $\mathcal{I}$.

Proof. The proof proceeds in two steps. First, one shows a counterpart of Lemma 1.1 in [Wil], namely, the following result: suppose $X \subset L^{1}(\mathbb{G})$ is a closed subspace and $\mathcal{F} \subset \mathrm{P}^{u}(\mathbb{G})$ is a closed convex semigroup such that $I_{\omega} \subset X$ for $\omega \in \mathcal{F}$, and that for each finite subset $A \subset X$ and $\varepsilon>0$ one can find $\omega_{A, \varepsilon} \in \mathcal{F}$ such that, for each $f \in A$, we have $d\left(f, I_{\omega_{A, \varepsilon}}\right)<\varepsilon$. Then, $X=I_{\omega}$ for some $\omega \in \mathcal{F}$. The proof follows line by line as in [Wil, Lemma 1.1]. Second, one uses this fact to prove that every chain in $\mathcal{I}$ has an upper bound, and concludes by the Kuratowski-Zorn lemma.

Theorem 3.4. Assume that $L^{1}(\mathbb{G})$ is separable. Consider the following list of conditions:

(i) $\mathbb{G}$ is coamenable.

(ii) $\mathbb{G}$ is amenable.

(iii) For each $\omega \in \mathrm{P}^{u}(\mathbb{G})$, the right ideal $I_{\omega}$ admits a bounded left approximate identity.

(iv) The collection $\mathcal{I}:=\left\{I_{\omega} \mid \omega \in \mathrm{P}^{u}(\mathbb{G})\right\}$ admits a unique maximal element $I_{\max }$. 
Then, the following implicationslequivalences hold: (ii) $\Longleftrightarrow$ (iv), (i) $\Longrightarrow$ (iii), and (i) + (ii) $\Longleftrightarrow$ (iii) + (iv). Moreover, if (iv) holds, then $I_{\max }=L_{0}^{1}(\mathbb{G})$; in particular, (iv) holds if and only if the augmentation ideal belongs to $\mathcal{I}$.

Proof. We begin by proving the last statement. Suppose then that (iv) holds and we have the largest ideal $I_{\max } \in \mathcal{I}$. By Lemma 3.2 we have

$$
I_{\max }^{\perp}=\left\{x \in L^{\infty}(\mathbb{G}) \mid \forall_{\omega \in \mathrm{P}^{u}(\mathbb{G})} L_{\omega}(x)=x\right\} .
$$

Suppose then that $x \in L^{\infty}(\mathbb{G})$ and $L_{\omega}(x)=\omega(1) x$ for all $\omega \in L^{1}(\mathbb{G})$. This is equivalent to $\Delta(x)=1 \otimes x$. As is well known, this holds if and only if $x \in \mathbb{C} 1$ (see for example Result 5.13 of $[\mathrm{KV}]$ ) and $I_{\max }=L_{0}^{1}(\mathbb{G})$.

The implication (ii) $\Longrightarrow$ (iv) follows now from the forward implication of Theorem 4.2 of $\left[\mathrm{KNR}_{1}\right]$. Further (iv) $\Longrightarrow$ (ii) follows as in the backward implication of Theorem 4.2 of $\left[\mathrm{KNR}_{1}\right]$, although the latter was formulated only for $\omega \in \mathrm{P}(\mathbb{G})$. Again, for completeness we sketch the proof. Suppose that (iv) holds, that is, there is $\omega \in \mathrm{P}^{u}(\mathbb{G})$ such that $I_{\omega}=L_{0}^{1}(\mathbb{G})$; equivalently, Fix $L_{\omega}=\mathbb{C} 1$. Pick a state $\mu \in L^{1}(\mathbb{G})$ and consider normal states $\omega_{n}:=S_{n}(\omega) \star \mu, n \in \mathbb{N}$. Let $\gamma \in S\left(L^{\infty}(\mathbb{G})\right)$ be the weak* limit point of the sequence $\left(\mu_{n}\right)_{n \in \mathbb{N}}$ along some free ultrafilter. Then, for every $x \in L^{\infty}(\mathbb{G})$ we have $R_{\gamma}(x) \in \operatorname{Fix} L_{\omega}=\mathbb{C} 1$ so that we can define a state $\gamma^{\prime}$ on $L^{\infty}(\mathbb{G})$ by the formula $\gamma^{\prime}(x) 1=R_{\gamma}(x), x \in L^{\infty}(\mathbb{G})$. One can then check that $\gamma=\gamma^{\prime}$, and in fact $\gamma$ is a left invariant mean on $L^{\infty}(\mathbb{G})$.

The implication (i) $\Longrightarrow$ (iii) follows as in the intro to [Wil]. Indeed, we fix $\omega \in \mathrm{P}^{u}(\mathbb{G})$. The fact that $\mathbb{G}$ is coamenable implies that $L^{1}(\mathbb{G})$ admits a bounded approximate identity, say $\left(u_{\lambda}\right)_{\lambda \in \Lambda}$. Then, one can check that the double-indexed net $\left(u_{\lambda}-S_{n}(\omega) \star u_{\lambda}\right)_{\lambda \in \Lambda, n \in \mathbb{N}}$ is a bounded left approximate identity in $I_{\omega}$.

The implication (iii) +(iv) $\Longrightarrow$ (i) +(ii) follows from Proposition 16 of $\left[\mathrm{HNR}_{1}\right]$; formally, the formulation in $\left[\mathrm{HNR}_{1}\right]$ requires the two-sided bounded approximate identity in $L_{0}^{1}(\mathbb{G})$, but as $L_{0}^{1}(\mathbb{G})$ is an involutive Banach algebra (with the involution given by composing with the unitary antipode), the existence of a one-sided bounded approximate identity implies that the two-sided one also exists.

\section{FIXED POINTS IN $C_{0}(\mathbb{G})$}

In this section, we consider the case when there are non-zero fixed points in $C_{0}(\mathbb{G})$. This only happens in the following cases: $\mathbb{G}$ is compact or $\omega$ is degenerate.

Lemma 4.1. Let $\omega \in \mathrm{M}^{u}(\mathbb{G})_{1}$ and suppose that the Cesàro averages $S_{n}(\omega)$ do not converge to 0 weak $k^{*}$ on $C_{0}^{u}(\mathbb{G})$. Then, the weak $k^{*}$ limit $\tilde{\omega}:=\mathrm{w}^{*}-\lim _{n \rightarrow \infty} S_{n}(\omega)$ exists, and $\tilde{\omega}$ is a non-zero contractive idempotent in $M^{u}(\mathbb{G})$.

Proof. Since the Cesàro sums $S_{n}(\omega)$ do not converge to 0 weak* on $C_{0}^{u}(\mathbb{G})$, there is some free ultrafilter $p$ such that the limit $\tilde{\omega}_{p}$ is non-zero on $C_{0}^{u}(\mathbb{G})$. By Lemma 1.7, $\tilde{\omega}_{p}$ is a contractive idempotent in $M^{u}(\mathbb{G})$. We shall show that in fact $\tilde{\omega}_{p}$ is the weak* limit of the sequence $\left(S_{n}(\omega)\right)_{n=1}^{\infty}$ by showing that every subnet of $\left(S_{n}(\omega)\right)_{n=1}^{\infty}$ has a subnet converging to $\tilde{\omega}_{p}$ (see [Wi, Exercise 11D.(c)]). 
Since $\left(S_{n}(\omega)\right)_{n=1}^{\infty}$ is bounded, every subnet of $\left(S_{n}(\omega)\right)_{n=1}^{\infty}$ has a subnet converging weak* to some $\tilde{\omega}_{q}$, where $q$ is some free ultrafilter. Let $a \in C_{0}^{u}(\mathbb{G})$ be arbitrary. Since $\tilde{\omega}_{p} \star \omega=\tilde{\omega}_{p}$, it follows that $\omega^{\star k}\left(L_{\tilde{\omega}_{p}}^{u}(a)\right)=\tilde{\omega}_{p}(a)$. Therefore, $S_{n}(\omega)\left(L_{\tilde{\omega}_{p}}^{u}(a)\right)=\tilde{\omega}_{p}(a)$, and so $\tilde{\omega}_{q}\left(L_{\tilde{\omega}_{p}}^{u}(a)\right)=\tilde{\omega}_{p}(a)$. It follows from Lemma 1.7 that $\tilde{\omega}_{q}$ is also a non-zero contractive idempotent in $M^{u}(\mathbb{G})$.

Since $\tilde{\omega}_{q} \in M^{u}(\mathbb{G})$, we have by Proposition 1.4 that

$$
\tilde{\omega}_{p}=\tilde{\omega}_{q} \circ L_{\tilde{\omega}_{p}}^{u}=\tilde{\omega}_{p} \star \tilde{\omega}_{q}=\tilde{\omega}_{p} \circ R_{\tilde{\omega}_{q}}^{u} .
$$

But

$$
\tilde{\omega}_{p} \circ R_{\tilde{\omega}_{q}}^{u}(a)=p-\lim S_{n}(\omega)\left(R_{\tilde{\omega}_{q}}^{u}(a)\right)=\tilde{\omega}_{q}(a)
$$

as $\omega \star \tilde{\omega}_{q}=\tilde{\omega}_{q}$. Consequently, $\tilde{\omega}_{q}=\tilde{\omega}_{p}$, as required.

Remark 4.2. The final condition in the following result may be interpreted as a weak analogue to "the subgroup generated by the support of $\omega$ is compact". Note that classically the closed subsemigroup generated by some set in a topological group is compact if and only if the closed subgroup generated by the set is compact (because a compact semigroup with cancellation laws is a group). See also Corollary 4.6.

The first result covers the general, possibly degenerate case.

Proposition 4.3. Let $\omega \in \mathrm{M}^{u}(\mathbb{G})_{1}$. Then, the following are equivalent:

(i) Cesàro sums $S_{n}(\omega)$ do not converge to 0 weak* on $C_{0}^{u}(\mathbb{G})$.

(ii) For some (equivalently for every) free ultrafilter $p$, we have that the functional $\tilde{\omega}_{p}:=p-\lim S_{n}(\omega)$ is a non-zero contractive idempotent in $M^{u}(\mathbb{G})$.

(iii) $L_{\omega}^{u}$ has a non-zero fixed point in $C_{0}^{u}(\mathbb{G})$.

(iv) $L_{\omega}$ has a non-zero fixed point in $C_{0}(\mathbb{G})$.

(v) There is a non-zero $\tau \in \mathrm{M}^{u}(\mathbb{G})$ such that $\tau \star \omega=\tau$.

(vi) $L_{\omega}$ has a non-zero fixed point in $L^{\infty}(\mathbb{G})$, and there exists e in $C_{0}^{u}(\mathbb{G})_{+}$such that $|\omega|^{\star k} \cdot e=e .|\omega|^{\star k}=|\omega|^{\star k}$ and ae $-a \in N_{|\omega|}$. for every $k=$ $1,2, \ldots$ and $a \in C_{0}^{u}(\mathbb{G})$.

The proof of this result will be based on the following lemma. For $v \in M(\mathbb{C})$, we define

$$
R_{v}: C_{0}(\mathbb{G}) \rightarrow C_{0}^{u}(\mathbb{G}), \quad R_{v}(a)=(\mathrm{id} \otimes v) \Delta_{S}(a) .
$$

This is possible because (1.1) implies that $\Delta_{S}(a) \in M\left(C_{0}^{u}(\mathbb{G}) \otimes C_{0}(\mathbb{G})\right)$ for every $a \in C_{0}(\mathbb{G})$.

Lemma 4.4. Let $\omega \in \mathrm{M}^{u}(\mathbb{G})_{1}$. If $L_{\omega}$ has a non-zero fixed point in $C_{0}(\mathbb{G})$, then there exists $v \in C_{0}(\mathbb{G})$ such that $\Delta_{u}^{(k)}(v)-v^{\otimes k+1} \in N_{|\omega|^{\otimes k+1}}$ and $\omega^{\star k}=v \cdot|\omega|^{\star k}$ for every $k \in \mathbb{N}$. Moreover, $|\omega|^{\star k}\left(a v^{*} v\right)=|\omega|^{\star k}\left(v^{*} v a\right)=|\omega|^{\star k}(a)$ for every $a \in C_{0}^{u}(\mathbb{G})$ and $k \in \mathbb{N}$. 
Proof. Suppose that $L_{\omega}$ has a non-zero fixed point $x$ in $C_{0}(\mathbb{G})$. Then, we may choose $v \in \mathrm{M}(\mathbb{G})_{1}$ such that $v(x)=\|x\|$. Then, $v=R_{v}\left(x^{*}\right) / \overline{v(x)} \in C_{0}^{u}(\mathbb{G})$ satisfies $\|v\| \leq 1$ and $\omega\left(v^{*}\right)=1$. Repeating the argument of Lemma 2.6, we see that this $v$ satisfies the statement (we may put in the proof there $\phi_{n}=v$ so that $v_{n}=v$ for all $n \in \mathbb{N}$ ).

Proof of Proposition 4.3. The following hold: (i) $\Longrightarrow$ (ii) (for every free ultrafilter): This follows from Lemma 4.1.

(ii) (for a fixed free ultrafilter) $\Longrightarrow$ (iii): This is clear because every element in $L_{\tilde{\omega}_{p}}^{u}\left(C_{0}(\mathbb{G})\right)$ is a fixed point of Fix $L_{\omega}^{u}$. (Note also that the map $\mu \mapsto L_{\mu}^{u}$ is injective.)

(iii) $\Longrightarrow$ (i): Let $a \in C_{0}^{u}(\mathbb{G})$ be a non-zero fixed point of $L_{\omega}^{u}$. Consider, then, $v \in \mathrm{M}^{u}(\mathbb{G})$ such that $v(a) \neq 0$. Then, we have

$$
\omega^{\star n}\left(R_{v}(a)\right)=v\left(L_{\omega^{\star n}}(a)\right)=v\left(L_{\omega}^{n}(a)\right)=v(a),
$$

and it follows that $S_{n}\left(R_{v}(a)\right)=v(a) \neq 0$ for every $n \in \mathbb{N}$.

(ii) (for a fixed free ultrafilter) $\Longrightarrow$ (iv): If $\tilde{\omega}_{p}$ is a non-zero contractive idempotent in $M^{u}(\mathbb{G})$, then $L_{\tilde{\omega}_{p}}\left(C_{0}(\mathbb{G})\right)=$ Fix $L_{\omega} \cap C_{0}(\mathbb{G})$ is non-zero (see Lemma 2.2).

(iv) $\Longrightarrow$ (i): This implication is similar to “(iii) $\Longrightarrow$ (i)" when we take $v \in M(\mathbb{G})$ and when $R_{v}: C_{0}(\mathbb{G}) \rightarrow C_{0}^{u}(\mathbb{G})$ is the map defined before Lemma 4.4.

(ii) (for a fixed free ultrafilter) $\Longrightarrow$ (v): Take $\tau=\tilde{\omega}_{p}$.

(v) $\Longrightarrow$ (iii): If $a \in C_{0}^{u}(\mathbb{G})$ is such that $L_{\tau}^{u}(a) \neq 0$, then the latter is a fixed point of $L_{\omega}^{u}$ as $L_{\omega}^{u} \circ L_{\tau}^{u}=L_{\tau \star \omega}^{u}=L_{\tau}^{u}$.

(iv) $\Longrightarrow$ (vi): Suppose that $L_{\omega}$ has a non-zero fixed point $x \in C_{0}$ ( $\mathbb{G}$ ). Then, we can apply Lemma 4.4 to obtain a suitable $v \in C_{0}^{u}(\mathbb{G})$. As (iv) implies (i), we know that the limit $\tilde{\omega}:=\mathrm{w}^{*}-\lim _{n \rightarrow \infty} S_{n}(\omega)$ exists by Lemma 4.1. We shall then show that $|\tilde{\omega}|(a)=|\omega|^{\sim}(a)$ for every $a \in C_{0}^{u}(\mathbb{G})$. Note that $\omega^{\star k}(a)=|\omega|^{\star k}(a v)$ by Lemma 4.4, and hence

$$
\tilde{\omega}(a)=\lim \frac{1}{n} \sum_{k=1}^{n} \omega^{\star k}(a)=\lim \frac{1}{n} \sum_{k=1}^{n}|\omega|^{\star k}(a v)=|\omega|^{\sim}(a v) .
$$

Recalling that $|\omega|^{\star k}\left(a v^{*} v\right)=|\omega|^{\star k}(a)$ for all $k \in \mathbb{N}$, it follows similarly that $\tilde{\omega}\left(a v^{*}\right)=|\omega|^{\sim}(a)$. These identities imply that $\|\tilde{\omega}\|=\left\||\omega|^{\sim}\right\|$. Moreover, by the Cauchy-Schwarz inequality,

$$
|\tilde{\omega}(a)|^{2} \leq|\omega|^{\sim}\left(a a^{*}\right)|\omega|^{\sim}\left(v^{*} v\right) \leq|\omega|^{\sim}\left(a a^{*}\right) .
$$

It now follows from the uniqueness of absolute value (Proposition III.4.6 of [Tak]) that $|\tilde{\omega}|=|\omega|^{\sim}$. 
Since $x \in C_{0}(\mathbb{G})$ is a fixed point of $L_{\omega}$, we have $L_{\tilde{\omega}}(x)=x$. Since $\tilde{\omega}$ is a contractive idempotent in $\mathrm{M}^{u}(\mathbb{G})$ it follows from Lemma 3.1 of [NSSS] (see also equation (2.5) of [Kas]) that

$$
x x^{*}=L_{\tilde{\omega}}(x) L_{\tilde{\omega}}(x)^{*}=L_{|\bar{\omega}|}\left(x L_{\tilde{\omega}}(x)^{*}\right)=L_{|\bar{\omega}|}\left(x x^{*}\right) .
$$

Pick $v \in \mathrm{M}(\mathbb{G})_{+}$, so $v\left(x x^{*}\right)=\left\|x x^{*}\right\|$. Then, $R_{v}\left(x x^{*}\right) \in C_{0}^{u}(\mathbb{G})$ is a fixed point of $L_{|\tilde{\omega}|}^{u}$ and $|\tilde{\omega}|\left(R_{v}\left(x x^{*}\right)\right)=v\left(x x^{*}\right)$. Set $e=R_{v}\left(x x^{*}\right) / v\left(x x^{*}\right) \in C_{0}^{u}(\mathbb{G})$. Since $|\tilde{\omega}|$ is an idempotent state in $\mathrm{M}^{u}(\mathbb{G})$ and $e \in L_{|\tilde{\omega}|}^{u}\left(C_{0}^{u}(\mathbb{G})\right)$, we have that $e$ is in the multiplicative domain of $|\tilde{\omega}|$ by Lemma 2.5 of $\left[\mathrm{SaS}_{1}\right]$.

Since $|\tilde{\omega}|=|\omega|^{\sim}$, we have for any $k \in \mathbb{N}$ that

$$
L_{|\omega|^{\star k}}\left(x x^{*}\right)=L_{|\omega|^{\star k}}\left(L_{|\tilde{\omega}|}\left(x x^{*}\right)\right)=L_{|\tilde{\omega}| \star|\omega| \star k}\left(x x^{*}\right)=L_{|\tilde{\omega}|}\left(x x^{*}\right)=x x^{*},
$$

and so $|\omega|^{\star k}(e)=1$. Hence, $1=|\omega|^{\star k}(e)^{2} \leq|\omega|^{\star k}\left(e^{2}\right) \leq 1$ (by CauchySchwarz), and it follows that $e$ is also in the multiplicative domain of $|\omega|^{\star k}$ for every $k \in \mathbb{N}$. Now, for every $a \in C_{0}^{u}(\mathbb{G})$ and $k \in \mathbb{N}$,

$$
\begin{aligned}
& |\omega|^{\star k}\left((a e-a)^{*}(a e-a)\right) \\
& \quad=|\omega|^{\star k}\left(e a^{*} a e\right)-|\omega|^{\star k}\left(a^{*} a e\right)-|\omega|^{\star k}\left(e a^{*} a\right)+|\omega|^{\star k}\left(a^{*} a\right)=0
\end{aligned}
$$

because $e$ is in the multiplicative domain of $|\omega|^{\star k}$. Therefore, ae $-a \in N_{|\omega|^{\star k}}$. (vi) $\Longrightarrow$ (i): Fix a free ultrafilter $p$ and let $\tilde{\omega}_{p} \in \mathrm{RUC}^{u}(\mathbb{G})^{*}$ be the weak* limit of $S_{n}(\omega)$ along $p$. Since $L_{\omega}$ has a non-zero fixed point in $L^{\infty}(\mathbb{G})$, there is, by Theorem 2.3, $x \in \mathrm{RUC}^{u}(\mathbb{G})$ such that $\tilde{\omega}_{p}(x) \neq 0$. Let $v \in C_{0}^{u}(\mathbb{G})^{* *}$ be as in Lemma 2.6. It follows from (vi) that $|\omega|^{\star k} . e=|\omega|^{\star k}$ on $C_{0}^{u}(\mathbb{G})^{* *}$ for every $k \in \mathbb{N}$. Therefore,

$$
\begin{aligned}
\tilde{\omega}_{p}(e x) & =p-\lim S_{n}(\omega)(e x)=p-\lim S_{n}(|\omega|)(e x v)=p-\lim S_{n}(|\omega|)(x v) \\
& =p-\lim S_{n}(\omega)(x)=\tilde{\omega}_{p}(x) \neq 0 .
\end{aligned}
$$

Since ex $\in C_{0}^{u}(\mathbb{G})$, (i) holds.

The above theorem has an immediate corollary, which can be also proved directly.

Corollary 4.5. If $\omega \in \mathrm{M}^{u}(\mathbb{G})_{1}$ and $\omega^{\star n} \rightarrow 0$ in the weak $k^{*}$ topology of $M^{u}(\mathbb{G})$, then Fix $L_{\omega} \cap C_{0}(\mathbb{G})=\{0\}$.

In the non-degenerate case, Proposition 4.3 can be used to completely characterise the fixed points in $C_{0}(\mathbb{G})$. Note that the second statement can be deduced from Theorem 2.7, and the analogous fact for $\omega$ being positive.

Corollary 4.6. Suppose that $\omega \in \mathrm{M}^{u}(\mathbb{G})_{1}$ is non-degenerate. If $\mathbb{G}$ is not compact, then Fix $L_{\omega} \cap C_{0}(\mathbb{G})=\{0\}$. If $\mathbb{G}$ is compact, then Fix $L_{\omega}$ is either $\{0\}$ or $\mathbb{C} u$ where $u$ is a group-like unitary in $C(\mathbb{G})$. 
Proof. For non-degenerate $\omega$, the element $e$ in statement (iv) of Proposition 4.3 is a positive right identity, and hence an identity. Therefore, $\mathbb{G}$ is necessarily compact if $L_{\omega}$ has a non-zero fixed point in $C_{0}(\mathbb{G})$.

Now, suppose $\mathbb{G}$ is compact. By Theorem 2.7 and Theorem 2.3, if Fix $L_{\omega} \neq$ $\{0\}$ there is a group-like unitary $u \in C(\mathbb{G})$ such that $\operatorname{Fix} L_{\omega}=\operatorname{Fix} L_{|\omega|} u$. By $\left[\mathrm{KNR}_{1}\right.$, Theorem 3.6], Fix $L_{|\omega|}=\mathbb{C} 1$.

Finally, we note that for discrete quantum groups and positive functionals the above corollary can be strengthened: in $\left[\mathrm{Ka}_{1}\right.$, Theorem 2] it is shown that if $\mathbb{G}$ is discrete and infinite (in other words non-compact) and $\omega \in \mathrm{P}^{u}(\mathbb{G})$ is nondegenerate, then for every $x \in C_{0}(\mathbb{G})$ we have $L_{\omega^{\star n}}(x) \stackrel{n \rightarrow \infty}{\longrightarrow} 0$.

\section{FiXed Points in $L_{p}(\mathbb{G})$ FOR Tracial HaAR Weights}

Yau showed in [Yau] that any harmonic function $f \in L_{p}(M)$ on a complete manifold $M$ is constant, for any $p \in(1, \infty)$. Motivated by this result, Chu [Chu] introduced and studied the space of $L_{p}$-fixed points of the convolution operator $L_{\omega}$ for $p \in[1, \infty)$. The main result of [Chu] states that if $\omega$ is an adapted probability measure, then any such fixed point must be a constant function (see [Chu, Theorem 3.12, Corollary 3.14]).

A quantum group version of Chu's result has been obtained by Kalantar in $\left[\mathrm{Ka}_{2}\right]$. More specifically, let $\mathbb{G}$ be a locally compact quantum group with tracial (left) Haar weight, $p \in[1, \infty)$, and $\omega \in P(\mathbb{G})$ a non-degenerate quantum probability measure. Consider the space of $\omega$-harmonic vectors in the noncommutative $L_{p}$-space $L_{p}(\mathbb{G})$ :

$$
H_{\omega}^{p}(\mathbb{G}):=\left\{f \in L_{p}(\mathbb{G}) \mid L_{\omega} f=f\right\} .
$$

If $\mathbb{G}$ is non-compact, then $H_{\omega}^{p}(\mathbb{G})=\{0\}\left[\mathrm{Ka}_{2}\right.$, Theorems 2.4, 2.6]. If $\mathbb{G}$ is compact, then $H_{\omega}^{p}(\mathbb{G})=\mathbb{C} 1\left[\mathrm{Ka}_{2}\right.$, Theorem 2.8].

We will now generalise these results to our setting, extending the context from $M(\mathbb{G})$ to $\mathrm{M}^{u}(\mathbb{G})$ and allowing non-positive quantum measures. We begin by outlining the construction of the action of the convolution operators on the noncommutative $L_{p}$-spaces, with $p \in[1, \infty)$. Recall that we denote by $\varphi$ the tracial left Haar weight, and by $\psi=\varphi R$ the right Haar weight, where $R$ is the unitary antipode of $L^{\infty}(\mathbb{G})$. We write $L_{p}(\mathbb{G})$ and $\tilde{L}_{p}(\mathbb{G})$ for the non-commutative $L_{p}$ spaces associated with $\varphi$ and $\psi$, respectively. Recall that these are defined as the completions of $\mathcal{M}_{\varphi}$ and $\mathcal{M}_{\psi}$ under the norms $\|x\|_{p}=\varphi\left(|x|^{p}\right)^{1 / p}$ and $\|x\|_{p}=$ $\psi\left(|x|^{p}\right)^{1 / p}$, respectively; here,

$$
\mathcal{M}_{\varphi}:=\operatorname{lin}\left\{x \in L^{\infty}(\mathbb{G})^{+} \mid \varphi(x)<\infty\right\} .
$$

For $p, q \in(1, \infty)$ with $1 / p+1 / q=1$, the spaces $L_{p}(\mathbb{G})$ and $\tilde{L}_{q}(\mathbb{G})$ are the duals of each other, via

$$
\langle a, b\rangle=\varphi(a R(b))=\psi(R(a) b) \quad\left(a \in L_{p}(\mathbb{G}), b \in \tilde{L}_{q}(\mathbb{G})\right)
$$


(cf. $\left[\mathrm{Ka}_{2}\right.$, p. 3972]). Recall we denote by $L^{1}(\mathbb{G})$ the predual of $L^{\infty}(\mathbb{G})$; the spaces $L^{1}(\mathbb{G})$ and $L_{1}(\mathbb{G})$ are isometrically isomorphic via the map $\Phi: L_{1}(\mathbb{G}) \rightarrow L^{1}(\mathbb{G})$ such that for $x \in \mathcal{M}_{\varphi}$ and $y \in L^{\infty}(\mathbb{G})$ we have $\Phi(x)(y)=\varphi(x y)$.

The convolution action of $M(\mathbb{G})$ on $L_{p}(\mathbb{G})$ is defined by complex interpolation; for $\omega \in P(\mathbb{G})$ this is discussed in in [Ka르. Section 2] (cf. also page 19, in particular Lemma 4.3, in $[\mathrm{BrR}]$ ). Below we will outline what is needed to extend this to $\omega \in M^{u}(\mathbb{G})$.

Begin by recalling the argument appearing in $\left[\mathrm{Ka}_{2}\right]$ : if $\omega \in M(\mathbb{G})_{+}$, then the convolution operator $L_{\omega}: L^{\infty}(\mathbb{G}) \rightarrow L^{\infty}(\mathbb{G})$ preserves the weight $\phi$ (so also the space $\mathcal{M}_{\varphi}$ ). Further, Kalantar verifies in $\left[\mathrm{Ka}_{2}\right]$ that we have the following equality:

$$
\Phi \circ L_{\omega}(z)=(\omega \circ R) \star \Phi(z) \quad\left(z \in \mathcal{M}_{\varphi}\right) .
$$

This implies that in fact $L_{\omega}$ yields (by continuous extension) a bounded map on $L_{1}(\mathbb{G})$, and hence by complex interpolation on all the spaces $L_{p}(\mathbb{G})$. Further, the above formula is true (simply by linearity and continuity) for all $\omega \in M(\mathbb{G})$ and $z \in L_{1}(\mathbb{G})$. A similar procedure shows that the right convolution operators $R_{\omega}$ act boundedly on $\tilde{L}_{q}(\mathbb{G})$.

In additio, Kalantar shows in the proof of $\left[\mathrm{Ka}_{2}\right.$, Theorem 2.2] that the following is true for all $p \in(1, \infty), \omega \in P(\mathbb{G}), f \in L_{p}(\mathbb{G})$, and $g \in \tilde{L}_{q}(\mathbb{G})$ :

$$
\left\langle L_{\omega}(f), g\right\rangle=\left\langle f, R_{\omega}(g)\right\rangle .
$$

Before we extend these formulas to operators associated with $\omega \in \mathrm{M}^{u}(\mathbb{G})$, we need to recall that for every $p \in(1, \infty)$, each of the subspaces $L_{1}(\mathbb{G}) \cap L_{p}(\mathbb{G})$ and $L^{\infty}(\mathbb{G}) \cap L_{p}(\mathbb{G})$ is dense in $L_{p}(\mathbb{G})$ (with similar statements holding for $L_{p}(\mathbb{G})$ ). Finally, note one more easy property: the span of $\left\{L_{v}(z) \mid v \in L^{1}(\mathbb{G}), z \in \mathcal{M}_{\varphi}\right\}$ is dense in $L_{1}(\mathbb{G})$ (and naturally the span of $\left\{R_{v}(z) \mid v \in L^{1}(\mathbb{G}), z \in \mathcal{M}_{\psi}\right\}$ is dense in $L_{1}(\mathbb{G})$ ). Indeed, it suffices to use the formula (5.1), the fact that $R$ is an isometry on $L^{\infty}(\mathbb{G})$, and finally the fact that the linear span of $L^{1}(\mathbb{G}) \star L^{1}(\mathbb{G})$ is dense in $L^{1}(\mathbb{G})$.

Lemma 5.1. Let $\omega \in \mathrm{M}^{u}(\mathbb{G}), p \in[1, \infty)$. Then, the operator

$$
L_{\omega}: L^{\infty}(\mathbb{G}) \rightarrow L^{\infty}(\mathbb{C})
$$

defines by restriction/continuous extension a bounded operator on $L_{p}(\mathbb{G})$ (to be denoted by the same symbol). Further, we have the following equalities:

$$
\begin{aligned}
\Phi \circ L_{\omega}(z) & =\left(\omega \circ R^{u}\right) \star \Phi(z), & & z \in L_{1}(\mathbb{G}), \\
\left\langle L_{\omega}(f), g\right\rangle & =\left\langle f, R_{\omega}(g)\right\rangle, & & f \in L_{p}(\mathbb{G}), g \in \tilde{L}_{q}(\mathbb{G}) .
\end{aligned}
$$

Proof. We follow the line of argument in [ $\left.\mathrm{Ka}_{2}\right]$. Note we can (and do) assume that $\omega \in \mathrm{P}^{u}(\mathbb{G})$ and then argue by linearity. The fact that $L_{\omega}$ preserves the left 
Haar weight is Lemma 3.4 of $\left[\mathrm{KNR}_{1}\right]$. In the next step, we show that the formula (5.1) holds for $z$ in a dense subset of $L_{1}(\mathbb{G})$. Indeed, take $z=L_{v}\left(z^{\prime}\right)$, where $z^{\prime} \in \mathcal{M}_{\varphi}$ and $v \in L^{1}(\mathbb{G})$. Then, we have

$$
\begin{aligned}
\Phi \circ L_{\omega}(z) & =\Phi \circ L_{\omega}\left(L_{v}\left(z^{\prime}\right)\right)=\Phi \circ L_{v \star \omega}\left(z^{\prime}\right)=((\nu \star \omega) \circ R) \star \Phi\left(z^{\prime}\right) \\
& =\left(\left(\omega \circ R_{u}\right) \star(\nu \circ R)\right) \star \Phi\left(z^{\prime}\right)=\left(\omega \circ R_{u}\right) \star\left((v \circ R) \star \Phi\left(z^{\prime}\right)\right) \\
& =\left(\omega \circ R_{u}\right) \star \Phi \circ L_{v}\left(z^{\prime}\right)=\left(\omega \circ R_{u}\right) \star \Phi(z),
\end{aligned}
$$

where in the third and the sixth equalities we used (5.1), and in the fourth one (1.2). Now, as the span of $L_{v}\left(z^{\prime}\right)$ with $z^{\prime} \in \mathcal{M}_{\varphi}$ and $v \in L^{1}(\mathbb{G})$ is dense in $L_{1}(\mathbb{G})$, we deduce that $L_{\omega}$ restricts/extends to a bounded map on the whole $L_{1}(\mathbb{G})$, and further (5.3) holds for all $x \in L_{1}(\mathbb{G})$. Now that we have established that $L_{\omega}$ is bounded on $L_{1}(\mathbb{G})$ (as well as on $L^{\infty}(\mathbb{G})$ ), it follows by complex interpolation that $L_{\omega}$ is bounded also on $L_{p}(\mathbb{G})$ for $p \in(1, \infty)$. Similarly, $R_{\omega}$ is bounded on all $\tilde{L}_{q}(\mathbb{G})$.

Consider now the second statement, and assume first that $p=1$. Suppose that $f=L_{v}\left(f^{\prime}\right)$ for some $v \in L^{1}(\mathbb{G})$ and $f^{\prime} \in L_{1}(\mathbb{G})$ and $g \in L^{\infty}(\mathbb{G})$. We compute

$$
\begin{aligned}
\left\langle L_{\omega}(f), g\right\rangle & =\left\langle L_{\omega}\left(L_{v}\left(f^{\prime}\right)\right), g\right\rangle=\left\langle L_{v \star \omega}\left(f^{\prime}\right), g\right\rangle=\left\langle f^{\prime}, R_{v \star \omega}(g)\right\rangle \\
& =\left\langle f^{\prime}, R_{v}\left(R_{\omega}(g)\right)\right\rangle=\left\langle L_{v}\left(f^{\prime}\right), R_{\omega}(g)\right\rangle=\left\langle f, R_{\omega}(g)\right\rangle,
\end{aligned}
$$

where in the third and fifth equalities we used (5.2). By continuity, (5.4) follows (for $p=1$ ).

Let then $p \in(1, \infty)$. As each duality is given by computing the trace on the respective (strong) products of measurable operators, we have by the above that

$$
\left\langle L_{\omega}(f), g\right\rangle=\left\langle f, R_{\omega}(g)\right\rangle
$$

for all $f \in L_{p}(\mathbb{G}) \cap L_{1}(\mathbb{G}), g \in \tilde{L}_{q}(\mathbb{G}) \cap L^{\infty}(\mathbb{G})$. Then, the general statement follows by first approximating a general $f \in L_{p}(\mathbb{G})$ by $f_{i} \in L_{p}(\mathbb{G}) \cap L_{1}(\mathbb{G})$ and then a general $g \in \tilde{L}_{q}(\mathbb{G})$ by $g_{i} \in \tilde{L}_{q}(\mathbb{G}) \cap L^{\infty}(\mathbb{G})$. This uses the continuity of the operators $L_{\omega}$ and $R_{\omega}$ on, respectively, $L_{p}(\mathbb{G})$ and $\tilde{L}_{q}(\mathbb{G})$.

We are ready for the main result of this section.

Theorem 5.2. Let $\mathbb{G}$ be a locally compact quantum group with tracial (left) Haar weight, let $p \in[1, \infty)$, and let $\omega \in M(\mathbb{G})_{1}$ be non-degenerate.

(i) Assume $\mathbb{G}$ is non-compact. Then, $H_{\omega}^{p}(\mathbb{G})=\{0\}$.

(ii) Assume $\mathbb{G}$ is compact and co-amenable. If $L_{\omega}$ has a non-zero fixed point in $L_{p}(\mathbb{G})$, then $H_{\omega}^{p}(\mathbb{G})=\mathbb{C} u$ where $u$ is a group-like unitary in $C(\mathbb{G})$.

Proof. (i) Case 1: $p>1$. Let $f \in H_{\omega}^{p}(\mathbb{G})$, and $g \in \tilde{L}_{q}(\mathbb{G}), g=R_{v}\left(g^{\prime}\right)$ for some $v \in L^{1}(\mathbb{G})$ and $g^{\prime} \in \tilde{L}_{q}(\mathbb{G})$. Then, the element $\Omega_{f, g^{\prime}} \in L_{\infty}(\mathbb{G})$ defined 
through $\left\langle\Omega_{f, g}, h\right\rangle=\left\langle L_{h}(f), g^{\prime}\right\rangle$ (with $h \in L^{1}(\mathbb{G})$ ) belongs to $C_{0}(\mathbb{G})$ (cf. $\left[\mathrm{Ka}_{2}\right.$, Proof of Theorem 2.4]) (note that the latter argument assumes $p \in(1,2]$, but it can be modified to work for all $p>1$ by using the density result obtained in [Mas, Theorem 8] to approximate $f$ and $g)$. Note also that $S_{n}(\omega) \rightarrow 0\left(w^{*}\right)$, since otherwise, by our Proposition $4.3, L_{\omega}$ would have a non-zero fixed point in $C_{0}(\mathbb{G})$, which would contradict the non-compactness of $\mathbb{G}$, in view of our Corollary 4.6. Thus we obtain, using twice (5.4),

$$
\begin{aligned}
\langle f, g\rangle & =\left\langle L_{S_{n}(\omega)} f, g\right\rangle=\left\langle f, R_{S_{n}(\omega)}(g)\right\rangle=\left\langle f, R_{S_{n}(\omega)}\left(R_{v}\left(g^{\prime}\right)\right)\right\rangle \\
& =\left\langle f, R_{S_{n}(\omega) \star v}\left(g^{\prime}\right)\right\rangle=\left\langle L_{S_{n}(\omega) \star v}(f), g^{\prime}\right\rangle \\
& =\left(S_{n}(\omega) \star v\right)\left(\Omega_{f, g^{\prime}}\right)=S_{n}(\omega)\left((\operatorname{id} \otimes v) \Delta\left(\Omega_{f, g^{\prime}}\right)\right) \stackrel{n \rightarrow \infty}{\longrightarrow} 0,
\end{aligned}
$$

whence $f=0$ (since $g$ was an arbitrary element of a dense subset of $\tilde{L}_{q}(\mathbb{G})$ ).

Case 2: $p=1$. Let $f \in H_{\omega}^{1}(\mathbb{G})$. Note that, as in Case 1, we have that $S_{n}(\omega) \rightarrow 0\left(w^{*}\right)$. By (5.3) we have for any $n \in \mathbb{N}$ the following equality:

$$
\Phi(f)=\Phi \circ L_{S_{n}(\omega)}(f)=\left(S_{n}(\omega) \circ R_{u}\right) \star \Phi(f) .
$$

Thus, for all $a \in C_{0}(\mathbb{G})$,

$$
\begin{aligned}
\Phi(f)(a) & =\left(\left(S_{n}(\omega) \circ R_{u}\right) \star \Phi(f)\right)(a) \\
& =\left(S_{n}(\omega) \circ R_{u}\right)((\operatorname{id} \otimes \Phi(f)) \Delta(a)) \\
& =S_{n}(\omega)\left(R_{u}((\operatorname{id} \otimes \Phi(f)) \Delta(a))\right) \stackrel{n \rightarrow \infty}{\longrightarrow} 0,
\end{aligned}
$$

so that $\Phi(f)=0$ and of course $f=0$.

(ii) Since $\mathbb{G}$ is compact, we have $L^{\infty}(\mathbb{G}) \subseteq L_{p}(\mathbb{G}) \subseteq L_{1}(\mathbb{G})$. Let $f \in H_{\omega}^{p}(\mathbb{G}) \backslash\{0\}$. As $\mathbb{G}$ is coamenable, we have a bounded approximate identity $\left(e_{\lambda}\right)_{\lambda \in \Lambda}$ in $L^{1}(\mathbb{G})$. For all $n \in \mathbb{N}$, choose $e_{\lambda_{n}}$ such that $\left\|\Phi(f) \star e_{\lambda_{n}}-\Phi(f)\right\|_{1}<1 / n$. Since $L^{\infty}(\mathbb{G})$ is dense in $L_{1}(\mathbb{G})$, we can further find $x_{n} \in L^{\infty}(\mathbb{G})$ with $\left\|\Phi\left(x_{n}\right)-e_{\lambda_{n}}\right\|_{1}<$ $1 /\left(n\|f\|_{1}\right)$. Hence, for all $n \in \mathbb{N}$ we obtain

$$
\begin{aligned}
& \left\|\Phi(f) \star \Phi\left(x_{n}\right)-\Phi(f)\right\|_{1} \\
& \quad \leq\left\|\Phi(f) \star \Phi\left(x_{n}\right)-\Phi(f) \star e_{\lambda_{n}}\right\|_{1}+\left\|\Phi(f) \star e_{\lambda_{n}}-\Phi(f)\right\|_{1}<\frac{2}{n},
\end{aligned}
$$

so $f_{n}:=\Phi^{-1}\left(\Phi(f) \star \Phi\left(x_{n}\right)\right) \stackrel{n \rightarrow \infty}{\longrightarrow} f$ in $L_{1}(\mathbb{G})$. We can now exploit the formula (5.1) to see that

$$
\Phi(f) \star \Phi\left(x_{n}\right)=((\Phi(f) \circ R) \circ R) \star \Phi\left(x_{n}\right)=\Phi\left(L_{\Phi(f) \circ R}\left(x_{n}\right)\right) .
$$

Thus, $f_{n}=L_{\Phi(f) \circ R}\left(x_{n}\right) \in L^{1}(\mathbb{G}) \star L^{\infty}(\mathbb{G})=R U C(\mathbb{G})=C(\mathbb{G})$. Finally,

$$
L_{\omega} \circ L_{\Phi(f) \circ R}\left(x_{n}\right)=L_{(\Phi(f) \circ R) \star \omega}\left(x_{n}\right),
$$


and using the (5.1) once again we check that

$$
(\Phi(f) \circ R) \star \omega=((\omega \circ R) \star \Phi(f)) \circ R=\Phi\left(L_{\omega}(f)\right) \circ R=\Phi(f) \circ R .
$$

As $f_{n} \rightarrow f \neq 0$, we have $f_{n} \neq 0$ for $n$ large enough, so that $L_{\omega}$ has a non-zero fixed point in $C(\mathbb{G})$. Thus, our Corollary 4.6 implies, since $\mathbb{G}$ is compact, that Fix $L_{\omega}=\mathbb{C} u$, where $u$ is a group-like unitary in $C(\mathbb{G})$. Thus, $f_{n} \in \mathbb{C} u$ for $n$ large enough, whence $f \in \mathbb{C} u$. Hence, we have $H_{\omega}^{p}(\mathbb{G}) \subseteq \mathbb{C} u$. The reverse inclusion is clear as $u \in \operatorname{Fix} L_{\omega} \subseteq L^{\infty}(\mathbb{G}) \subseteq L^{p}(\mathbb{G})$.

\section{Classical Case}

In this section we discuss the classical case, that is, the situation where $G$ is a locally compact group.

Given a probability measure $\omega \in \operatorname{Prob}(G)$, we let $S_{\omega}$ and $G_{\omega}$ denote, respectively, the closed semigroup and group generated by supp $\omega$. We say that $\omega$ is non-degenerate (or irreducible) if $S_{\omega}=G$, and adapted if $G_{\omega}=G$, so that non-degeneracy implies adaptedness, but the converse implication does not hold. Given a general $\omega \in \mathrm{M}(G)$ we will call it non-degenerate (respectively, adapted) if $|\omega|$ is non-degenerate (respectively, adapted). It is easy to see that this notion of non-degeneracy coincides with the one introduced earlier. In the non-degenerate case, Theorem 2.7 says that if $L_{\omega}$ has a non-zero fixed point in $\operatorname{LUC}(G)$, then there is $\chi \in \hat{G}$ such that $\omega=\chi|\omega|$ and Fix $L_{\omega}=\left(\operatorname{Fix} L_{|\omega|}\right) \bar{\chi}$. The following theorem addresses this result in the degenerate situation.

Theorem 6.1. Suppose that $G$ is a locally compact group and that $\omega \in \mathrm{M}(G)_{1}$. If $L_{\omega}$ has a non-zero fixed point in $\operatorname{LUC}(G)$, then there is a continuous character $\chi: S_{|\omega|} \rightarrow \mathbb{T}$ such that $\omega=\chi|\omega|$.

Proof. Let $f \in \operatorname{LUC}(G)$ be a non-zero fixed point of $L_{\omega}$. Let $\tilde{f}$ denote the extension of $f$ to the LUC-compactification $G^{\mathrm{LUC}}$. Then, there exists $x \in G^{\mathrm{LUC}}$ such that $\|f\|=|\tilde{f}(x)|$. Multiplying $f$ by a scalar, we may and shall assume that $1=\tilde{f}(x)=\|f\|$.

Write $\omega=u|\omega|$ where $u$ is a unimodular, measurable function. With $x$ as above, which can be viewed also as an element of $\operatorname{LUC}(G)^{*}$, we define a function $x \odot f$ by

$$
(x \odot f)(s)=\left\langle x, \ell_{s} f\right\rangle=\tilde{f}(s x) \quad(s \in G)
$$

where $s x$ is defined using the multiplication on $G^{\mathrm{LUC}}$. Then, $x \odot f \in \operatorname{LUC}(G)$, and for any $n \in \mathbb{N}$, if we choose a net $\left(t_{i}\right)_{i \in \mathcal{I}}$ of elements of $G$ convergent to $x$ inside $G^{\mathrm{LUC}}$, we have

$$
\begin{aligned}
\left\langle\omega^{\star n}, x \odot f\right\rangle & =\left\langle\omega^{\star n} \cdot x, f\right\rangle=\lim _{i \in \mathcal{I}}\left\langle\omega^{\star n} \cdot t_{i}, f\right\rangle=\lim _{i \in \mathcal{I}}\left\langle\omega^{\star n}, r_{t_{i}} f\right\rangle \\
& =\lim _{i \in \mathcal{I}}\left(L_{\omega}^{n} f\right)\left(t_{i}\right)=\left\langle x, L_{\omega}^{n} f\right\rangle=\langle x, f\rangle,
\end{aligned}
$$


where we first used the definition of the product in $\operatorname{LUC}(G)^{*}$ and then the fact that $\omega^{\star n}$, as a measure, belongs to the topological centre of $\operatorname{LUC}(G)^{*}$ (see, e.g., [Won]). Hence, letting $S=\operatorname{supp}|\omega|$, we have that

$$
\tilde{f}(x)=\int_{S^{\times n}} \tilde{f}\left(s_{1} \ldots s_{n} x\right) u\left(s_{1}\right) \ldots u\left(s_{n}\right) \mathrm{d}|\omega|^{\times n}\left(s_{1}, \ldots, s_{n}\right) .
$$

Since $|\tilde{f}|$ attains its maximum at $x$, and $\tilde{f}(x)=1$,

$$
\tilde{f}\left(s_{1} \ldots s_{n} x\right) u\left(s_{1}\right) \ldots u\left(s_{n}\right)=\tilde{f}(x)=1
$$

for $|\omega|^{\times n}$ almost every $\left(s_{1}, \ldots, s_{n}\right)$. Now, $\overline{\chi(s)}=\tilde{f}(s x)$ defines a bounded continuous function $\chi$ on $G$. Taking $n=1$ above, we see that

$$
\chi(s)=u(s) \quad \text { for }|\omega| \text {-almost every } s \text {, and hence } \chi(S) \subseteq \mathbb{T} .
$$

For arbitrary $n$, we then see that

$$
\chi\left(s_{1} \ldots s_{n}\right)=u\left(s_{1}\right) \ldots u\left(s_{n}\right) \text { for }|\omega|^{\times n} \text {-almost every }\left(s_{1}, \ldots, s_{n}\right),
$$

while the $n=1$ case implies $u\left(s_{1}\right) \ldots u\left(s_{n}\right)=\chi\left(s_{1}\right) \ldots \chi\left(s_{n}\right)$ for $|\omega|^{\times n}$-almost every $\left(s_{1}, \ldots, s_{n}\right)$. Since $\chi$ is continuous, we conclude that

$$
\chi\left(s_{1} \ldots s_{n}\right)=\chi\left(s_{1}\right) \ldots \chi\left(s_{n}\right) \quad \text { for every } n \in \mathbb{N} \text { and }\left(s_{1}, \ldots, s_{n}\right) \in S^{\times n} .
$$

We let $S^{n} \subseteq G$ denote the set of products from $S^{\times n}$. An obvious induction shows that $\chi$ is multiplicative on the semigroup $S_{|\omega|}^{\prime}=\bigcup_{n=1}^{\infty} S^{n}$, and hence, by continuity, $X$ is multiplicative on its closure $S_{|\omega|}$.

Notice that if $|\omega|$ in the last theorem is non-degenerate, then the character $\chi$ with the desired properties is defined on the whole of $G$. In such a situation, if $f \in \operatorname{Fix} L_{|\omega|}$, then it is clear that $f \bar{\chi} \in \operatorname{Fix} L_{\omega}$. In other cases, there is no evident analogue of this fact. For any closed subsemigroup $S$ of $G$ and continuous character $x: S \rightarrow \mathbb{T}$, we let

$$
\operatorname{LUC}_{X, S}(G)=\{f \in \operatorname{LUC}(G) \mid f(s t)=\overline{\chi(s)} f(t) \text { for } s \in S, t \in G\} .
$$

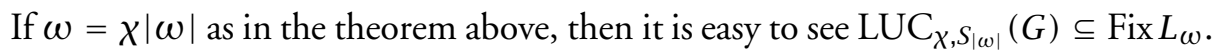
If $\omega=|\omega|$ is a probability measure, then in many cases (e.g., $G$ abelian ([ChD]) or $G$ is SIN and $\omega$ is non-degenerate [Jaw]) we have the Choquet-Deny theorem: Fix $L_{\omega}=\mathrm{LUC}_{1, S_{|\omega|}}(G)$.

Suppose we are given a closed subsemigroup $S$ densely generating a locally compact group $H$. Except in the case of abelian groups, it is not evident how to extend a character $\chi_{0}: S \rightarrow \mathbb{T}$ to a character on $H$. Even in the abelian case it is not clear that the extension can be assumed continuous if $\chi_{0}$ is continuous. 
Lemma 6.2. Let $S$ be a closed subsemigroup of $G$, let $H$ be the smallest closed subgroup of $G$ containing $S$, and let $X_{0}: S \rightarrow \mathbb{T}$ be a continuous character. If there exists a non-zero bounded continuous function $f$ such that $f(s t)=\overline{X_{0}(s)} f(t)$ for all $s \in S, t \in G$, then $\chi_{0}$ extends to a continuous character $\chi$ on $H$. Furthermore, $f(s t)=\overline{\chi(s)} f(t)$ for all $s \in H, t \in G$.

Proof. Set $U_{f}=\{t \in G \mid f(t) \neq 0\}$. Let $s \in S$ and $t \in U_{f}$. We have that $\overline{\chi_{0}(s)} f=\ell_{s} f$ (left translation), and hence $\ell_{s^{-1}} f=\chi_{0}(s) f$, which implies that

$$
\overline{\chi_{0}(s)}=\frac{f(s t)}{f(t)} \quad \text { and } \quad \chi_{0}(s)=\frac{f\left(s^{-1} t\right)}{f(t)} \quad \text { so } \quad\langle S\rangle U_{f} \subset U_{f},
$$

where $\langle S\rangle$ is the subgroup generated (algebraically) by $S$. We fix some $t_{0} \in U_{f}$ and define $\chi: G \rightarrow \mathbb{C}$ by

$$
\overline{\chi(s)}=\frac{f\left(s t_{0}\right)}{f\left(t_{0}\right)}
$$

so that $X$ is continuous. Then, for $s, s^{\prime}$ in $S \cup S^{-1}$, we have that

$$
\overline{\chi\left(s s^{\prime}\right)}=\frac{f\left(s s^{\prime} t_{0}\right)}{f\left(s^{\prime} t_{0}\right)} \frac{f\left(s^{\prime} t_{0}\right)}{f\left(t_{0}\right)}=\overline{\chi(s) \chi\left(s^{\prime}\right)} .
$$

A simple induction then shows that $\chi$ is multiplicative on $\langle S\rangle=\bigcup_{n=1}^{\infty}\left(S \cup S^{-1}\right)^{n}$, so also on $H$. Similarly, we see that $\ell_{s} f=\overline{\chi(s)} f$ on $\langle S\rangle$, and hence also on $H$, which ends the proof.

We consider below two cases in which the Choquet-Deny theorem always holds in the context of probability measures, and show that it also holds in the more general framework of contractive measures we consider here. For the abelian case we adapt the remarkably simple proof of [Rau], and for the weakly almost periodic case we adapt a proof from [Tem], which Tempel'man attributes to RyllNardzewski. Below, $W A P(G)$ denotes the space of weakly almost periodic functions on $G$; recall also the notation introduced in (6.1). hold:

Theorem 6.3. Let $G$ be a locally compact group and $\omega \in \mathrm{M}(G)_{1}$. The following

(i) If $G$ is abelian and $L_{\omega}$ has a non-zero fixed point in $\operatorname{LUC}(G)$, then there is a continuous character $\chi: G|\omega| \rightarrow \mathbb{T}$ such that $\omega=\chi|\omega|$ and Fix $L_{\omega}=$ $\operatorname{LUC}_{X, G|\omega|}(G)$.

(ii) If $L_{\omega}$ has a non-zero fixed point in $\operatorname{WAP}(G)$ then there is a continuous character $\chi: G_{|\omega|} \rightarrow \mathbb{T}$ such that $\omega=\chi|\omega|$ and $\operatorname{Fix} L_{\omega} \cap \operatorname{WAP}(G)=$

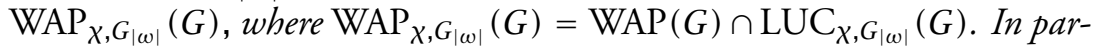
ticular, we have that $\left.\chi \in \operatorname{WAP}(G)\right|_{G|\omega|}$.

Proof. In either case above, Theorem 6.1 provides a character $\chi: S_{|\omega|} \rightarrow \mathbb{T}$ for which $\omega=\chi|\omega|$. We will proceed to show for each case that the fixed points 
of $L_{\omega}$ are contained in the set $\operatorname{LUC}_{X, S_{|\omega|}}(G)$. Note that Lemma 6.2 shows that we may deem from the beginning $X$ to be a character on $G_{|\omega|}$.

(i) We let $f \in \operatorname{Fix} L_{\omega} \backslash\{0\}$ and write $S=\operatorname{supp}|\omega|$. Define for $n \in \mathbb{N}$ and $t \in G$

$$
\begin{aligned}
g_{n}(t)=\int_{S^{\times n}} & \mid \chi\left(s_{1} \ldots s_{n}\right) f\left(s_{1} \ldots s_{n} t\right) \\
& \quad-\left.\chi\left(s_{1} \ldots s_{n-1}\right) f\left(s_{1} \ldots s_{n-1} t\right)\right|^{2} \mathrm{~d}|\omega|^{\times n}\left(s_{1}, \ldots, s_{n}\right) .
\end{aligned}
$$

Expanding the integrand, exploiting the fact that $G$ is abelian (so that $s_{1} \ldots s_{n} t=$ $\left.s_{n} s_{1} \ldots s_{n-1} t\right)$ and that $f \in \operatorname{Fix} L_{\omega}$, we see that

$$
g_{n}=L_{|\omega| \star n}\left(|f|^{2}\right)-L_{|\omega|^{\star(n-1)}}\left(|f|^{2}\right) .
$$

Hence, the telescopic series $\sum_{n=1}^{\infty} g_{n}$ converges (as increasing and bounded), so that $\lim _{n \rightarrow \infty} g_{n}=0$. On the other hand, the Cauchy-Schwarz inequality tells us that for each $t \in G$,

$$
\begin{aligned}
& \int_{S^{\times(n-1)}} \mid \chi\left(s_{1} \ldots s_{n}\right) f\left(s_{1} \ldots s_{n} t\right)- \\
& \quad-\left.\chi\left(s_{1} \ldots s_{n-1}\right) f\left(s_{1} \ldots s_{n-1} t\right)\right|^{2} \mathrm{~d}|\omega|^{\times(n-1)}\left(s_{1}, \ldots, s_{n-1}\right) \\
& \geq \mid \int_{S^{\times(n-1)}}\left[\chi\left(s_{1} \ldots s_{n}\right) f\left(s_{1} \ldots s_{n} t\right)\right. \\
& \left.\quad-\chi\left(s_{1} \ldots s_{n-1}\right) f\left(s_{1} \ldots s_{n-1} t\right)\right]\left.\mathrm{d}|\omega|^{\times(n-1)}\left(s_{1}, \ldots, s_{n-1}\right)\right|^{2} \\
& =\left|\chi\left(s_{n}\right) f\left(s_{n} t\right)-f(t)\right|^{2} .
\end{aligned}
$$

Hence, an application of the Fubini-Tonelli theorem shows that $g_{n} \geq g_{1}$. Thus, $g_{1}=0$, which tells us for any $t$ that $\chi(s) f(s t)=f(t)$ for $|\omega|$-almost every $s$, and hence, by continuity, for every $s \in S$. In other words, $\ell_{s} f=\overline{\chi(s)} f$ for $s \in S$. A simple induction shows this holds for $s \in \bigcup_{n=1}^{\infty} S^{n}$, and hence for all $s \in S_{|\omega|}$.

(ii) Let $f \in \operatorname{Fix} L_{\omega} \cap \operatorname{WAP}(G) \backslash\{0\}$. Consider the product probability space

$$
\left(\Omega, \mathcal{B}_{\infty}, P\right)=\left(G^{\infty}, \mathcal{B}_{\infty},|\omega|^{\times \infty}\right),
$$

where $\mathcal{B}_{\infty}$ is the $\sigma$-algebra generated by the sequence of $\sigma$-algebras given for each $n \in \mathbb{N}$ by

$$
\mathcal{B}_{n}=\sigma\left\langle B_{1} \times \cdots \times B_{n} \times G \times G \times \cdots: \text { each } B_{i} \text { is a Borel set in } G\right\rangle .
$$

Let $\mathcal{L}\left(\mathcal{B}_{n}\right)=L^{1}\left(\Omega, \mathcal{B}_{n}, P\right.$; WAP $\left.(G)\right)$ for each $n=1,2 \ldots, \infty$. We have conditional expectations $\mathbb{E}_{n}: \mathcal{L}\left(\mathcal{B}_{\infty}\right) \rightarrow \mathcal{L}\left(\mathcal{B}_{n}\right)$ given by

$$
\left[\mathbb{E}_{n}(\alpha)\right]\left(s_{1}, s_{2}, \ldots\right)=\int_{\Omega} \alpha\left(s_{1}, \ldots, s_{n-1}, s_{n}, s_{n+1}, \ldots\right) \mathrm{d} P\left(s_{n+1}, s_{n+2}, \ldots\right) .
$$


Define $\beta_{n}$ in $\mathcal{L}\left(\mathcal{B}_{n}\right)$ for $P$-almost every $\left(s_{1}, s_{2}, \ldots\right)$ by

$$
\beta_{n}\left(s_{1}, s_{2}, \ldots\right)=\chi\left(s_{1} \ldots s_{n}\right) \ell_{s_{n} \ldots s_{1}} f,
$$

and observe that $\left\|\beta_{n}\right\|_{L^{1}} \leq\|f\|_{\infty}$. Also, observe that we have

$$
\begin{aligned}
& \left\|\chi\left(s_{n}\right) \ell_{s_{n}} f-f\right\|_{\infty} \\
& \quad=\left\|\chi\left(s_{1} \ldots s_{n-1}\right) l_{s_{n-1} \ldots s_{1}}\left[\chi\left(s_{n}\right) \ell_{s_{n}} f-f\right]\right\|_{\infty} \\
& \quad=\left\|\left(\beta_{n}-\beta_{n-1}\right)\left(s_{1}, s_{2}, \ldots\right)\right\|_{\infty}
\end{aligned}
$$

for $P$-almost every $\left(s_{1}, s_{2}, \ldots\right)$. We compute, for any $n \geq 2, t \in G$ and $\left(s_{1}, s_{2}, \ldots\right)$ as above that

$$
\begin{aligned}
& \left(\left[\mathbb{E}_{n-1}\left(\beta_{n}\right)\right]\left(s_{1}, s_{2}, \ldots\right)\right)(t) \\
& \quad=\chi\left(s_{1} \ldots s_{n-1}\right) \ell_{s_{n-1} \ldots s_{1}} \int_{G} f\left(s_{n} t\right) \chi\left(s_{n}\right) \mathrm{d}|\omega|\left(s_{n}\right) \\
& \quad=\chi\left(s_{1} \ldots s_{n-1}\right) \ell_{s_{n-1} \ldots s_{1}} f(t)=\left(\beta_{n-1}\left(s_{1}, s_{2}, \ldots\right)\right)(t) .
\end{aligned}
$$

Hence, $\left(\beta_{n}\right)_{n=1}^{\infty}$ is a martingale in $\mathcal{L}\left(\mathcal{B}_{\infty}\right)$. Moreover, the essential range of each $\beta_{n}$ is contained in $\mathbb{T}\left\{l_{g} f \mid g \in G\right\}$, and therefore is relatively weakly compact as $f \in \operatorname{WAP}(G)$. Hence, by [Cha, , VIII Theorem 2], there is $\beta$ in $\mathcal{L}(\mathcal{B})$, where $(\Omega, \mathcal{B}, \bar{P})$ is the completion of $\mathcal{B}_{\infty}$, for which $\beta=\lim _{n \rightarrow \infty} \beta_{n}, \bar{P}$-almost everywhere, and for each $n \in \mathbb{N}$ we have $\beta_{n}=\mathbb{E}_{n}(\beta)$. By Theorem 1 in [Cha $]$, $\lim _{n \rightarrow \infty}\left\|\beta_{n}-\beta\right\|_{L^{1}}=0$. Thus, for $P$-almost every $\left(s_{1}, s_{2}, \ldots\right)$, we have, using (6.2), that

$$
\begin{aligned}
\int_{G}\left\|\chi\left(s_{n}\right) \ell_{s_{n}} f-f\right\|_{\infty} \mathrm{d}|\omega|\left(s_{n}\right) & =\int_{\Omega}\left\|\beta_{n}-\beta_{n-1}\right\|_{\infty} \mathrm{d} P \\
& =\left\|\beta_{n}-\beta_{n-1}\right\|_{L^{1}} \stackrel{n \rightarrow \infty}{\longrightarrow} 0,
\end{aligned}
$$

and hence $\int_{G}\left\|\chi(s) \ell_{s} f-f\right\|_{\infty} \mathrm{d}|\omega|(s)=0$, so $\ell_{s} f=\overline{\chi(s)} f$ for $|\omega|$-almost every $s$. As in the last part of (i) above, we see that $f \in \operatorname{WAP}_{\chi, S|\omega|}(G)$.

Notice that by taking right translations, we may assume our non-zero fixed point $f$ satisfies $f(e) \neq 0$. Thus, for $s$ in $G_{|\omega|}$ we must have $f(s)=\overline{\chi(s)} f(e)$, so that $\left.\bar{\chi} \in \operatorname{WAP}(G)\right|_{G_{|\omega|}}$. Clearly, the algebra $\left.\operatorname{WAP}(G)\right|_{G_{|\omega|}}$ of functions on $G_{|\omega|}$ is self-adjoint.

We comment that the proof of (i) shows that if $\omega=\chi|\omega|$ for a continuous character $\chi$ on $S_{|\omega|}$, then $\ell_{s} h=\overline{\chi(s)} h$ for $s$ in $S_{|\omega|}$ and every bounded Borelmeasurable function $h$.

If $G$ is a semisimple Lie group with finite center, then it is shown in [Vee] ([Cho] for $G=\mathrm{SL}_{2}(\mathbb{R})$ ) that for any non-compact closed one-parameter subgroup $R \cong \mathbb{R}$ we have $\left.\operatorname{WAP}(G)\right|_{R}=C_{0}(\mathbb{R}) \oplus \mathbb{C} 1$. Hence, there are many measures $\omega$ in $\mathrm{M}(G)_{1}$ for which Fix $L_{\omega} \cap \operatorname{WAP}(G)=\{0\}$. 
If we ask about the fixed points in $C_{0}(G)$, also here in the classical case we obtain a more refined result than Proposition 4.3.

Corollary 6.4. Let $G$ be a locally compact group and $\omega \in \mathrm{M}(G)_{1}$. Then, the following are equivalent:

(i) Fix $L_{\omega} \cap C_{0}(G) \neq\{0\}$.

(ii) $G_{|\omega|}$ is compact and there is a continuous character $\chi: G_{|\omega|} \rightarrow \mathbb{T}$ such that $\omega=\chi|\omega|$.

(iii) Cesàro sums, $S_{n}(\omega)$, do not converge weak $k^{*}$ to 0 .

In this case, we have that $\chi m_{G_{|\omega|}}=\mathrm{w}^{*}-\lim _{n \rightarrow \infty} S_{n}(\omega)$, and that

$$
\operatorname{Fix} L_{\omega} \cap C_{0}(G)=\operatorname{Fix} L_{\chi m_{G|\omega|}} \cap C_{0}(G)=L_{\chi m_{G|\omega|}}\left(C_{0}(G)\right) .
$$

In particular, if $G$ is compact and $|\omega|$ is adapted, then Fix $L_{\omega}=\mathbb{C} \bar{X}$.

Proof. The equivalence of (i) and (iii) is from Proposition 4.3. It follows from Theorem 6.3 that (i) is equivalent to (ii), where the compactness of $G_{|\omega|}$ follows from the fact that if $f$ is a non-zero element of $C_{0}(G) \cap \operatorname{WAP}_{\chi, G|\omega|}(G)$ (see the terminology introduced in the last theorem), then $|f|$ is constant on cosets of $G_{|\omega|}$. Since $\chi m_{G_{|\omega|}}$ is an idempotent, a rudimentary computation shows that

$$
\begin{aligned}
C_{0, \chi, G|\omega|}(G) & :=\left\{f \in C_{0}(G) \mid f(s t)=\overline{\chi(s)} f(t) \text { for } s \in G_{|\omega|}, t \in G\right\} \\
& =L_{\chi m_{G_{|\omega|} \mid}}\left(C_{0}(G)\right) \\
& =\operatorname{Fix} L_{\chi m_{G_{|\omega|}}} \cap C_{0}(G) .
\end{aligned}
$$

Finally, by Lemma $4.1, \tilde{\omega}=\mathrm{w}^{*}-\lim _{n \rightarrow \infty} S_{n}(\omega)$ is a non-zero contractive idempotent with $C_{0, \chi}, G_{|\omega|}(G)=$ Fix $L_{\tilde{\omega}} \cap C_{0}(G)$. Hence, by [Gre], we have $\bar{\omega}=$ $\theta m_{K}$ for some compact group $K$ and a continuous character $\theta: K \rightarrow \mathbb{T}$. But Fix $L_{\theta m_{K}} \cap C_{0}(G)=L_{\theta m_{K}}\left(C_{0}(G)\right)=C_{0, \theta, K}(G)$. We conclude that $K=G_{|\omega|}$ and $\theta=\chi$, and hence $\tilde{\omega}=\chi m_{G_{|\omega|}}$.

Note that, in the case when $\omega \in \operatorname{Prob}(G)$ and $G_{\omega}$ is compact (i.e. when any of the equivalent assumptions of Corollary 6.4 hold, we have that $m_{G_{\omega}}=$ $\mathrm{w}^{*}-\lim _{n \rightarrow \infty} S_{n}(\omega)$ for $\omega$ in $\operatorname{Prob}(G)$, as shown in [KI].

We finish with some observations generalising the results of this section to a more abstract context, and present one more application.

Now, let $E$ be a Banach space. A strong operator-continuous representation $\pi: G \rightarrow G \mathcal{L}(E)$ will be called essentially weakly almost periodic if there is a separating subspace $F \subseteq E^{*}$ such that

$$
M_{\pi, F}=\operatorname{span}\{\langle f, \pi(\cdot) x\rangle \mid x \in E, f \in F\} \subseteq \operatorname{WAP}(G) ;
$$

that is, the space generated by matrix coefficients of $\pi$ with $F$ consists of weakly almost periodic functions. Notice that the uniform boundedness principle shows 
that $\pi$ must have bounded range. If $\omega \in \mathrm{M}(G)$, then we define the operator $\pi(\omega)$ in terms of Bochner integrals: $\pi(\omega) x=\int_{G} \pi(s) x \mathrm{~d} \omega(s)$ for $x$ in $E$.

Corollary 6.5. Let $G$ be a locally compact group, let $\omega \in \mathrm{M}(G)_{1}$, and let $\pi: G \rightarrow G \mathcal{L}(E)$ be an essentially weakly almost periodic representation. If $\pi(\omega)$ has a non-zero fixed point, then there is a continuous character $\chi: G_{|\omega|} \rightarrow \mathbb{T}$ such that $\omega=\chi|\omega|$ and

$$
\text { Fix } \pi(\omega)=\left\{x \in E \mid \pi(s) x=\overline{\chi(s)} x \text { for all } s \text { in } G_{|\omega|}\right\} .
$$

In particular, we have that $\left.\bar{X} \in M_{\pi, F}\right|_{G_{|\omega|}}$, where $F$ is as in the definition of essential weak almost periodicity of $\pi$.

Proof. Let $\check{\pi}(s)=\pi\left(s^{-1}\right)$ for $s$ in $G$, so that $\check{\pi}$ is an anti-homomorphism. If $x \in E$ and $f \in F$, let $\pi_{f, x}=\langle f, \pi(\cdot) x\rangle$. We also let $\check{\omega}$ denote the unique measure which satisfies $\int_{G} g \mathrm{~d} \check{\omega}=\int_{G} \check{g} \mathrm{~d} \omega$, where $\check{\mathfrak{g}}(s)=g\left(s^{-1}\right)$ for $g$ in $\operatorname{WAP}(G)$. Notice that $\check{\pi}_{f, x}=\langle f, \check{\pi}(\cdot) x\rangle$. We then have for $t$ in $G$ that

$L_{\check{\omega}} \check{\pi}_{f, x}(t)=\int_{G}\left\langle f, \check{\pi}\left(s^{-1} t\right) x\right\rangle \mathrm{d} \omega(s)=\int_{G}\langle f, \check{\pi}(t) \pi(s) x\rangle \mathrm{d} \omega(s)=\check{\pi}_{f, \pi(\omega) x}(t)$.

Since $F$ is separating, it follows that, for $x \in E$, we have $x \in$ Fix $\pi(\omega)$ if and only if $\check{\pi}_{f, x} \in$ Fix $L_{\breve{\omega}} \cap \operatorname{WAP}(G)$ for every $f$ in $F$. Suppose that Fix $\pi(\omega) \neq\{0\}$. By Theorem 6.3 (ii), $\breve{\omega}=\bar{\chi}|\check{\omega}|=\bar{\chi}|\omega|^{\vee}$ for some continuous character $\chi$ on $G_{|\check{\omega}|}=G_{|\omega|}$, and hence $\omega=\chi|\omega|$. Furthermore, we have $\check{\pi}_{f, x} \in \operatorname{WAP}_{\bar{\chi}, G_{|\omega|}}(G)$ whenever $x \in$ Fix $\pi(\omega)$ and $f \in F$. Hence, for $x$ in $E$, we have $x \in \operatorname{Fix} \pi(\omega)$ exactly, when for $f$ in $F$ and $s$ in $G_{|\omega|}$ we have

$$
\langle f, \pi(s) x\rangle=\check{\pi}_{f, x}\left(s^{-1}\right)=\chi\left(s^{-1}\right) \check{\pi}_{f, x}(e)=\overline{\chi(s)} \pi_{f, x}(e)=\langle f, \overline{\chi(s)} x\rangle .
$$

Also, again since $F$ is separating, this happens exactly when $\overline{\chi(s)} x=\pi(s) x$. In this case, a variant of the last computation also shows that $\overline{\chi(s)} \pi_{f, x}(e)=\pi_{f, x}(s)$, so $\left.\bar{\chi} \in M_{\pi, F}\right|_{G_{|\omega|} \mid}$.

Finally consider the left regular representation

$$
\lambda_{p}: G \rightarrow G \mathcal{L}\left(L^{p}(G)\right), \quad \lambda_{p}(s) f(t)=f\left(s^{-1} t\right)
$$

for each $s \in G$ and almost every $t \in G$. As before, we let $H_{\omega}^{p}(G)=\operatorname{Fix} \lambda_{p}(\check{\omega})$. In the commutative setting (i.e., the case of locally compact groups), we gain the following improvement to Theorem 5.2.

Corollary 6.6. Let $G$ be a locally compact group, $p \in[1, \infty)$, and $\omega \in \mathrm{M}(G)_{1}$. Suppose that $H^{p}(G) \neq\{0\}$. Then, $G_{|\omega|}$ is compact, $\omega=\chi|\omega|$ for some continuous character $\chi: G_{|\omega|} \rightarrow \mathbb{T}$, and $H_{\omega}^{p}(G)=\operatorname{Ran} \lambda_{p}\left(\bar{\chi} m_{G_{|\omega|}}\right)$ where $m_{G_{|\omega|}}$ is the normalised Haar measure on $G_{|\omega|}$. In particular, if $\omega$ is adapted, then $H_{\omega}^{p}(G)=\mathbb{C} \bar{X}$. 
Proof. Letting $F=L^{p^{\prime}}(G)$ (dual space) for $p>1$, or $C_{0}(G)$ for $p=1$, we see that $M_{\lambda_{p}, F} \subseteq C_{0}(G) \subseteq \mathrm{WAP}(G)$. We can then appeal directly to Corollaries 6.5 and 6.4 .

\section{Mukherjea Condition and the Dual of the Classical Case}

We devote the last section to a discussion of possible generalizations in the "dual to classical" case of the following classical result due to Mukherjea [Muk] (see also [Der]), and draw several consequences.

Theorem 7.1. Let $G$ be a locally compact group and let $\omega \in \operatorname{Prob}(G)$. Then, the following are equivalent:

(i) Cesàro sums $S_{n}(\omega)$ converge to 0 weak* on $C_{0}(G)$.

(ii) Convolution iterates $\omega^{\star n}$ converge to 0 weak ${ }^{*}$ on $C_{0}(G)$.

(iii) The group $G_{\omega}$ is not compact.

The result is due to Derriennic (Theorem 8 of [Der]), who assumed that $\omega$ is adapted. However, the form above is equivalent since extension holds (i.e., $\left.C_{0}(G)_{G_{\omega}}=C_{0}\left(G_{\omega}\right)\right)$. Mukherjea established this result earlier in [Muk] under the second countability assumption.

In this section we consider the cocommutative case, that is, the case when $\mathbb{G}$ is the dual of a locally compact group $G$. In this case, $C_{0}^{u}(\mathbb{G})^{*}$ is identified with the Fourier-Stieltjes algebra $B(G)$ (see $[\mathrm{Eym}]$ ), which is the linear span of $P(G)$ - the cone of continuous positive definite functions on $G$. Moreover, in this case $L^{\infty}(\mathbb{G})$ is the group von Neumann algebra $\operatorname{VN}(G)$, the preadjoint of which is the Fourier algebra $A(G)$ (again, see [Eym]). As we will show below in Theorem 7.5, the analogous result of Theorem 7.1 holds in the dual case, if we interpret condition (iii) in the following way: the preimage of 1 with respect to the normalised function $\omega \in \mathrm{B}(G)^{+}$is not open.

We cannot hope to extend Theorem 7.1 to contractive functionals as the implication (ii) $\Longrightarrow$ (iii) can fail (there exist nilpotent contractive measures on compact groups), as can (i) $\Longrightarrow$ (ii) (consider simply $\omega=-\delta_{e}$ ). We will still see in Theorem 7.5 that there is a simple way to cut out such "pathological" examples.

Finally, note that in the classical context (iii) $\Longrightarrow$ (ii) holds for $\omega \in \mathrm{M}(G)_{1}$, as follows from the above result and a simple convolution estimate $\left|\omega^{\star n}\right| \leq|\omega|^{\star n}$, $n \in \mathbb{N}$.

For $\omega \in B(G)$, write $Z_{\omega}=\{s \in G \mid \omega(s)=1\}$.

Theorem 7.2. Suppose that $G$ is a locally compact group and $\omega \in \mathrm{B}(G)_{1}$. The following hold:

(i) If $Z_{\omega}$ is empty, then Fix $L_{\omega}=\{0\}$. This occurs in particular when $\|\omega\|<1$.

(ii) If $Z_{\omega}$ is not empty, then $Z_{\omega}=s H$ where $H$ is a closed subgroup of $G$ and $s \in G$. Moreover, Fix $L_{\omega}=\pi(s) \mathrm{VN}(H)$.

In particular, Fix $L_{\omega}$ is always a $W^{*}-$ sub-TRO of $\mathrm{VN}(G)$, that is, Fix $L_{\omega}$ is a weak*closed subspace that is closed under the ternary product $(x, y, z) \mapsto x y^{*} z$. 
Proof. (i) Suppose that $x \neq 0$ is a fixed point of $L_{\omega}$. Let $s \in \operatorname{supp} x$ (see [Eym, Définition 4.5] for the definition of supp $x$ ), and pick $f \in A(G)$ such that $f(s) \neq 0$. Now, $0=L_{f}\left(x-L_{\omega}(x)\right)=L_{f-f \omega}(x)$. Therefore, as $f-f \omega \in A(G)$, it follows from Proposition 4.4 of $[$ Eym] that $(f-f \omega)(s)=0$. Since $f(s) \neq 0$, we have that $\omega(s)=1$. Thus, if $Z_{\omega}=\varnothing$, then Fix $L_{\omega}=\{0\}$.

The latter statement is clear because if $\|\omega\|<1$, then $|\omega(s)|<1$ for every $s \in G$.

(ii) Let $s \in Z_{\omega}$ and define $\tau(t)=\omega(s t)$ for $t \in G$. Then, $\tau \in \mathrm{B}(G)_{1}$ and $\tau(e)=1$ so $\tau$ is a state. Let $\pi: G \rightarrow M\left(C^{*}(G)\right)$ be the natural map. For every $t \in Z_{\tau}$, also $t^{-1} \in Z_{\tau}$ as $\tau$ is positive definite and

$$
\tau\left(\pi(t)^{*} \pi(t)\right)=\tau\left(t^{-1} t\right)=1=\overline{\tau(t)} \tau(t)=\tau\left(\pi(t)^{*}\right) \tau(\pi(t)) .
$$

Hence, $\pi(t)$ is in the multiplicative domain of $\tau$ (viewed as a state on $M\left(C^{*}(G)\right)$ ), and it follows that $Z_{\tau}$ is closed under multiplication. Therefore, $H:=Z_{\tau}$ is a closed subgroup of $G$ and $Z_{\omega}=s Z_{\tau}=s H$.

Let $t \in G$. Then, $L_{\omega}(\pi(t))=\omega(t) \pi(t)=\pi(t)$ if and only if $t \in s H$. It follows that $\pi(s) \mathrm{VN}(H) \subseteq$ Fix $L_{\omega}$. Conversely, if $x \in \operatorname{Fix} L_{\omega}$, then the proof of the statement (i) shows that supp $x \subseteq Z_{\omega}$, and so Fix $L_{\omega} \subseteq \pi(s) \mathrm{VN}(H)$.

Corollary 7.3. Suppose $\omega \in \mathrm{B}(G)_{1}$ is non-degenerate. Then, $\operatorname{dim}\left(\operatorname{Fix} L_{\omega}\right) \leq 1$. Moreover, if Fix $L_{\omega}$ is non-zero, it contains a unitary.

Proof. We shall show that if $\omega$ is non-degenerate, then $Z_{|\omega|} \subset\{e\}$. Suppose otherwise, and pick $s \in Z_{|\omega|} \backslash\{e\}$. Then,

$$
\begin{aligned}
& |\omega|^{\star k}\left((\pi(s)-\pi(e))^{*}(\pi(s)-\pi(e))\right) \\
& \quad=2|\omega|^{\star k}(e)-|\omega|^{\star k}(s)-|\omega|^{\star k}\left(s^{-1}\right)=0
\end{aligned}
$$

for every $k \in \mathbb{N}$, which is in contradiction with non-degeneracy. The preceding theorem implies that if Fix $L_{\omega} \neq\{0\}$, then Fix $L_{\omega}=\pi(s) \mathbb{C}$ for some $s \in G$ as $Z_{|\omega|}=H=\{e\}$ (in the notation of that theorem). Now, $\pi(s)$ is the required unitary element.

Before we proceed with the main result of this section, we record below how Theorem 7.2 provides an answer to a question asked by Chu and Lau, who posed the following in [ChL, Remark 3.3.16]. Let $\omega \in B(G)_{1}$ be such that $Z_{\omega}$ is discrete and $A(G) / I_{\omega}$ has a bounded approximate identity (recall that $I_{\omega}$ denotes the pre-annihilator of Fix $L_{\omega}$ in $A(G)$; see Definition 3.1). Does Arens regularity of $A(G) / I_{\omega}$ imply finiteness of $Z_{\omega}$ ? We now show that this is indeed the case.

Theorem 7.4. Let $G$ be a locally compact group, and let $\omega \in B(G)_{1}$ be such that $Z_{\omega}$ is discrete. Assume that the Banach algebra $A(G) / I_{\omega}$ has a BAI and is Arens regular. Then, $Z_{\omega}$ is finite. 
Proof. By assumption, we are in the situation of [ChL, Proposition 3.3.15 (ii)]. Hence, $E:=I_{\omega}^{\perp}=$ Fix $L_{\omega}$ is, as a Banach space, isomorphic to a Hilbert space, so in particular $E$ is reflexive. We can assume that $Z_{\omega}$ is non-empty. By Theorem 7.2 (ii), we have $Z_{\omega}=s H$ and $E=\lambda_{s} \mathrm{VN}(H)$, where $H$ is a closed subgroup of $G$ and $s \in G$. Thus, the Banach space $\mathrm{VN}(H)$ is isomorphic to $E$, and hence reflexive. But a von Neumann algebra whose underlying Banach space is reflexive is finite dimensional (cf., e.g. [Li, Proposition 1.11.7]). Thus, $H$ is finite, whence $Z_{\omega}=s H$ is finite as well.

We now return to the main theme of the section.

Theorem 7.5. If $\omega \in B(G)$ such that $\|\omega\|=1$ and $Z_{\omega} \neq \varnothing$, then the following are equivalent:

(i) Cesàro sums $S_{n}(\omega)$ converge to 0 weak* on $C^{*}(G)$.

(ii) Convolution iterates $\omega^{\star n}$ converge to 0 weak $k^{*}$ on $C^{*}(G)$.

(iii) The set $Z_{\omega}$ is not open (equivalently, $Z_{\omega}$ is locally null). In particular, the above statements are equivalent when $\omega$ is a state.

Proof. Note first that by Theorem 7.2, the set $Z_{\omega}$ is a coset of a closed subgroup $H$ of $G$. Naturally, $Z_{\omega}$ is open if and only if $H$ is open, and locally null with respect to the left Haar measure of $G$ if and only if $H$ is locally null. Now, the fact that $H$ is not locally null is equivalent to $H$ being open is standard (and follows from a Steinhaus type theorem; see, e.g., Corollary 20.17 in $[\mathrm{HeR}])$. We now proceed with the proof of the main equivalences.

That (ii) $\Longrightarrow$ (i) is trivial.

To prove (i) $\Longrightarrow$ (iii), assume that (iii) is not true. Since $Z_{\omega}$ is open, there is a compactly supported continuous function $f$ on $G$ such that $\operatorname{supp} f \subseteq Z_{\omega}$ and $\int f(s) \mathrm{d} s=1$ (note that $Z_{\omega} \neq \varnothing$ by assumption). If $\pi: L^{1}(G) \rightarrow C^{*}(G)$ is the canonical map, then

$$
\omega^{\star k}(\pi(f))=\int f(s) \omega(s)^{k} \mathrm{~d} s=\int f(s) \mathrm{d} s=1
$$

for every $k \in \mathbb{N}$. Hence, $S_{n}(\omega)(\pi(f))=1$ for every $n \in \mathbb{N}$, and (i) is not true.

To prove (iii) $\Longrightarrow$ (ii), let $\omega$ be as in the assumptions of the theorem. The proof of Theorem 7.2 shows there exists $s \in Z_{\omega}$ and a state $\tau \in B(G)$ such that $\tau(t)=$ $\omega(s t), t \in G$; moreover, $Z_{\omega}=s Z_{\tau}$ (so that (iii) is equivalent to $Z_{\tau}$ being null). If $f \in L^{1}(G)$, then

$$
\begin{aligned}
& \omega^{\star n}(\pi(f))=\int_{G} \omega^{n}(t) f(t) \mathrm{d} t, \\
& \tau^{\star n}(\pi(f))=\int_{G} \omega^{n}(s t) f(t) \mathrm{d} t=\int_{G} \omega^{n}(t) f\left(s^{-1} t\right) \mathrm{d} t .
\end{aligned}
$$


This implies that (ii) is equivalent to the convolution iterates $\tau^{\star n}$ converging to 0 weak* on $C^{*}(G)$.

The discussion above implies we can assume without loss of generality that $\omega$ is a state. There is then a strongly continuous unitary representation $\pi: G \rightarrow B(\mathrm{H})$ on some Hilbert space $\mathrm{H}$ and a unit vector $\xi \in \mathrm{H}$ such that $\omega(g)=\langle\xi, \pi(g) \xi\rangle$, $g \in G$. Write $U:=\omega^{-1}(\mathbb{T})$. As for any $z \in \mathbb{T}$ and $g \in G$, we have

$$
\omega(s)=z \Longleftrightarrow z=\langle\xi, \pi(g) \xi\rangle \Longleftrightarrow \pi(g) \xi=z \xi,
$$

and it is easy to see $U$ is a closed subgroup of $G$ and $\left.\omega\right|_{U}: U \rightarrow \mathbb{T}$ is a homomorphism with kernel equal to $Z_{\omega}$. Thus, $\left.\omega\right|_{U}=\chi \circ q$, where $q: U \rightarrow U / Z_{\omega}$ is the quotient map and $\chi \in \widehat{U / Z_{\omega}}$. Further, $U / Z_{\omega} \cong \omega(U) \subset \mathbb{T}$.

Let $f \in L^{1}(G)$. If $U$ is not open, then $\mu_{G}(U)=0$, as argued in the beginning of the proof, and we have

$$
\omega^{\star n}(\pi(f))=\int_{G \backslash U} \omega^{n}(t) f(t) \mathrm{d} t \stackrel{n \rightarrow \infty}{\longrightarrow} 0
$$

by the dominated convergence theorem. We may then assume that $U$ is open; and then $U / Z_{\omega}$ cannot be a finite subgroup of $\mathbb{T}$ (as $Z_{\omega}$ is assumed not open). Hence, $U / Z_{\omega} \cong \mathbb{T}$, and $X \neq 1$. By the standard integration formula, we have

$$
\begin{aligned}
\int_{U} \omega^{n}(t) f(t) \mathrm{d} t & =\int_{U / Z_{\omega}}\left(\int_{Z_{\omega}} \omega(s t)^{n} f(s t) \mathrm{d} \mu_{Z_{\omega}}(t)\right) \mathrm{d} \mu_{U / Z_{\omega}}\left(s Z_{\omega}\right) \\
& =\int_{U / Z_{\omega}} \chi\left(s Z_{\omega}\right)^{n}\left(\int_{Z_{\omega}} f(s t) \mathrm{d} \mu_{Z_{\omega}}(t)\right) \mathrm{d} \mu_{U / Z_{\omega}}\left(s Z_{\omega}\right),
\end{aligned}
$$

and the latter tends to 0 by the Riemann-Lebesgue lemma-since the function $s Z_{\omega} \mapsto \int_{Z_{\omega}} f(s t) \mathrm{d} \mu_{Z_{\omega}}(t)$ is integrable. This ends the proof.

Note that the above theorem is related to Theorem 3 of [Ka3], which is on one hand only treating the positive-definite functions $\omega$, but on the other obtains a stronger notion of convergence in (ii), that is, the point-norm convergence of the convolution iterates.

Furthermore, we note that the equivalence of (i) and (iii) in Theorem 7.5 above also follows from the recent, independently obtained result in Proposition 2.5 of [Mus]. However, the equivalence with (ii) in Theorem 7.5 does not follow from [Mus], as the example of $u(t)=e^{i t}$ on $G=\mathbb{R}$ shows, which is covered by our Theorem 7.5, but does not satisfy the assumption of Proposition 4.4 (a) in [Mus] (cf. the remark after the proof of the latter).

Finally, we stress that the condition that $Z_{\omega}$ is not open is connected to the fact that the "support" of $\omega$ is non-compact. We see this clearly in the case where $G$ is abelian. 
Proposition 7.6. Let $G$ be a locally compact abelian group, and let $\mu \in \mathrm{M}(G)$, $\|\mu\|=1$. Then, $Z_{\hat{\mu}} \neq \varnothing$ if and only if $\mathrm{d} \mu / \mathrm{d}|\mu|=\chi \mu$-almost everywhere for some character $\chi \in \hat{G}$. Moreover, in that case $G_{\mu}$ is compact if and only if $Z_{\hat{\mu}}$ is open.

Proof. If $\chi \in Z_{\hat{\mu}}$, then

$$
1=\hat{\mu}(\chi)=\int \overline{\chi(s)} \mathrm{d} \mu(s)=\int \overline{\chi(s)} \frac{\mathrm{d} \mu}{\mathrm{d}|\mu|}(s) \mathrm{d}|\mu|(s),
$$

which implies that $\mathrm{d} \mu / \mathrm{d}|\mu|=\chi \mu$-almost everywhere (because $\|\mu\|=1$ ). The converse is trivial.

Moreover, if $\chi \in Z_{\hat{\mu}}$, then

$$
\begin{aligned}
\eta \in Z_{\hat{\mu}} & \Leftrightarrow 1=\int \overline{\eta(s)} X(s) \mathrm{d}|\mu|(s) \\
& \Leftrightarrow \bar{\eta} X=1 \quad \text { on } \operatorname{supp} \mu \\
& \Leftrightarrow \bar{\eta} X=1 \quad \text { on } G_{\mu} \\
& \Leftrightarrow \eta \in X G_{\mu}^{\perp} .
\end{aligned}
$$

Then, the second statement follows from the fact that $G_{\mu}$ is compact if and only if $G_{\mu}^{\perp}$ is open.

Note that the preceding two results imply Mukherjea's result for locally compact abelian groups.

Acknowledgements. Work on this article was initiated during the authors' Research in Pairs visit to the Mathematisches Forschungsinstitut Oberwolfach in August 2012. Many thanks are due to the MFO for providing ideal research conditions. The first author was partially supported by the National Science Center (NCN) (grant no. 2014/14/E/ST1/00525), and the second author by the Emil Aaltonen Foundation. Finally, the authors express their thanks to the referee for a careful reading of the paper and several useful comments.

\section{REFERENCES}

[BMS] G. A. BAgheri-BArdi, A. R. Medghalchi, And N. SPronk, Operator-valued convolution algebras, Houston J. Math. 36 (2010), no. 4, 1023-1036. MR2753732.

[BrR] M. BRANNAN AND Z. J. RUAN, $L_{p}$-representations of discrete quantum groups, J. Reine Angew. Math. 732 (2017), 165-210. http://dx.doi.org/10.1515/crelle-2014-0140. MR3717091.

[Cha 1 S. D. CHATTERJI, A note on the convergence of Banach-space valued martingales, Math. Ann. 153 (1964), 142-149. http://dx.doi.org/10.1007/BF01361182. MR161376.

[Cha 2 _ Les martingales et leurs applications analytiques, École d'Été de Probabilités: Processus Stochastiques (Saint Flour, 1971), Lecture Notes in Math., vol. 307, Springer, Berlin, 1973, pp. 27-164 (French). MR0448536.

[ChD] G. Choquet And J. Deny, Sur l'équation de convolution $\mu=\mu * \sigma$, C. R. Acad. Sci. Paris 250 (1960), 799-801 (French). MR119041. 
[Cho] C. CHOU, Weakly almost periodic functions and Fourier-Stieltjes algebras of locally compact groups, Trans. Amer. Math. Soc. 274 (1982), no. 1, 141-157. http://dx.doi.org/10. 2307/1999501. MR670924.

[Chu] C. H. CHU, Harmonic function spaces on groups, J. London Math. Soc. (2) 70 (2004), no. 1, 182-198. http://dx.doi.org/10.1112/S0024610704005265. MR2064757.

[ChL] C. H. ChU AND A. T. M. LAU, Harmonic Functions on Groups and Fourier Algebras, Lecture Notes in Mathematics, vol. 1782, Springer-Verlag, Berlin, 2002. http://dx.doi.org/ 10.1007/b83280. MR1914221.

[Coh] P. J. CoHen, On homomorphisms of group algebras, Amer. J. Math. 82 (1960), 213-226. http://dx.doi.org/10.2307/2372732. MR133398.

[Daw] M. DAWS, Completely positive multipliers of quantum groups, Internat. J. Math. 23 (2012), no. 12, 1250132, 23 pp. http://dx.doi.org/10.1142/S0129167X12501327. MR3019431.

[Der] Y. DERRIENNIC, Lois "zéro ou deux" pour les processus de Markov: Applications aux marches aléatoires, Ann. Inst. H. Poincaré Sect. B (N.S.) 12 (1976), no. 2, 111-129 (French, with English summary). MR0423532.

[Eym] P. EYMARD, L'algèbre de Fourier d'un groupe localement compact, Bull. Soc. Math. France 92 (1964), 181-236 (French). http://dx.doi.org/10.24033/bsmf.1607. MR228628.

[Gre] F. P. GREENLEAF, Norm decreasing homomorphisms of group algebras, Pacific J. Math. 15 (1965), no. 4, 1187-1219. http://dx.doi.org/10.2140/pjm.1965.15.1187. MR194911.

[Grn] U. Grenander, Probabilities on Algebraic Structures, John Wiley \& Sons, Inc., New YorkLondon; Almqvist \& Wiksell, Stockholm-Göteborg-Uppsala, 1963. MR0206994.

[HeR] E. HewiTT AND K. A. Ross, Abstract Harmonic Analysis Vol. I: Structure of Topological Groups, Integration Theory, Group Representations, 2nd ed., Grundlehren Der Mathematischen Wissenschaften [Fundamental Principles of Mathematical Sciences], vol. 115, SpringerVerlag, Berlin-New York, 1979. MR551496.

$\left[\mathrm{HNR}_{1}\right]$ Z. HU, M. NEUfANG, AND Z. J. RUAN, On topological centre problems and SIN quantum groups, J. Funct. Anal. 257 (2009), no. 2, 610-640. http://dx.doi.org/10.1016/j.jfa. 2009.02.004. MR2527031.

$\left[\mathrm{HNR}_{2}\right]$, Completely bounded multipliers over locally compact quantum groups, Proc. Lond. Math. Soc. (3) 103 (2011), no. 1, 1-39. http://dx.doi.org/10.1112/plms/pdq041. MR2812500.

[Jaw] W. JAWORSKI, Ergodic and mixing probability measures on [SIN] groups, J. Theoret. Probab. 17 (2004), no. 3, 741-759. http://dx.doi.org/10.1023/B: JOTP.0000040297. 84097. 57. MR2091559.

[JaN] W. JAWORSKI AND M. NEUfANG, The Choquet-Deny equation in a Banach space, Canad. J. Math. 59 (2007), no. 4, 795-827. http://dx. doi .org/10.4153/CJM-2007-034-4. MR2338234.

[JNR] M. JUnge, M. NEUfANG, AND Z. J. RUAN, A representation theorem for locally compact quantum groups, Internat. J. Math. 20 (2009), no. 3, 377-400. http://dx.doi.org/10. 1142/S0129167X09005285. MR2500076.

[Kai] V. A. KaImANOVICH, Boundaries of invariant Markov operators: The identification problem, Ergodic Theory of $\mathbb{Z}^{d}$ Actions (Warwick, 1993), London Math. Soc. Lecture Note Ser., vol. 228, Cambridge Univ. Press, Cambridge, 1996, pp. 127-176. http://dx.doi.org/ 10.1017/CB09780511662812.005. MR1411218.

[Ka 1 M. KalantaR, A limit theorem for discrete quantum groups, J. Funct. Anal. 265 (2013), no. 3, 469-473. http://dx.doi.org/10.1016/j.jfa.2013.05.014. MR3056713.

[Kaㄹ _ On harmonic non-commutative $L^{p}$-operators on locally compact quantum groups, Proc. Amer. Math. Soc. 141 (2013), no. 11, 3969-3976. http://dx.doi.org/10.1090/ S0002-9939-2013-11763-6. MR3091787. 
[Ka3] _ On iterated powers of positive definite functions, Bull. Aust. Math. Soc. 92 (2015), no. 3, 440-443. http://dx.doi.org/10.1017/S0004972715000490. MR3415620.

$\left[\mathrm{KNR}_{1}\right]$ M. KalantaR, M. NeUfang, AND Z. J. RUAN, Poisson boundaries over locally compact quantum groups, Internat. J. Math. 24 (2013), no. 3, 1350023, 21 pp. http://dx.doi .org/ 10.1142/S0129167X13500237. MR3048010.

$\left[\mathrm{KNR}_{2}\right] \longrightarrow$, Realization of quantum group Poisson boundaries as crossed products, Bull. Lond. Math. Soc. 46 (2014), no. 6, 1267-1275. http://dx.doi.org/10.1112/blms/bdu081. MR3291263.

[Kas] P. KASPRZAK, Shifts of group-like projections and contractive idempotent functionals for locally compact quantum groups, Internat. J. Math. 29 (2018), no. 13, 1850092, 19 pp. http://dx. doi.org/10.1142/S0129167X18500921. MR3894765.

[KI] Y. KAWADA AND K. ITÔ, On the probability distribution on a compact group. I, Proc. Phys.Math. Soc. Japan (3) 22 (1940), 977-998. MR3462.

[Kus] J. KUSTERMANS, Locally compact quantum groups in the universal setting, Internat. J. Math. 12 (2001), no. 3, 289-338. http://dx.doi.org/10.1142/S0129167X01000757. MR1841517.

[KV] J. Kustermans AND S. VAeS, Locally compact quantum groups, Ann. Sci. École Norm. Sup. (4) 33 (2000), no. 6, 837-934 (English, with English and French summaries). http: // dx.doi.org/10.1016/S0012-9593(00)01055-7. MR1832993.

[Li] B. R. LI, Introduction to Operator Algebras, World Scientific Publishing Co., Inc., River Edge, NJ, 1992. MR1194183.

[Mas] T. MASUDA, $L_{p}$-spaces for von Neumann algebra with reference to a faithful normal semifinite weight, Publ. Res. Inst. Math. Sci. 19 (1983), no. 2, 673-727. http://dx.doi.org/10. 2977/prims/1195182447. MR716971.

[Muk] A. MUKHERJEA, Limit theorems for probability measures on non-compact groups and semigroups, Z. Wahrscheinlichkeitstheorie und Verw. Gebiete 33 (1975/76), no. 4, 273-284. http://dx.doi.org/10.1007/BF00534779. MR400334.

[Mus] H. MustafayeV, Mean ergodic theorems for multipliers on Banach algebras, J. Fourier Anal. Appl. 25 (2019), no. 2, 393-426. http://dx.doi.org/10.1007/s00041-017-9587-x. MR3917951.

[NaT] Y. NAKAGAmi AND M. TAKeSAKI, Duality for Crossed Products of von Neumann Algebras, Lecture Notes in Mathematics, vol. 731, Springer, Berlin, 1979. MR546058.

[NSSS] M. Neufang, P. Salmi, A. SKalski, AND N. Spronk, Contractive idempotents on locally compact quantum groups, Indiana Univ. Math. J. 62 (2013), no. 6, 1983-2002. http: // dx.doi.org/10.1512/iumj.2013.62.5178. MR3205538.

[Rau] A. RAUGI, A general Choquet-Deny theorem for nilpotent groups, Ann. Inst. H. Poincaré Probab. Statist. 40 (2004), no. 6, 677-683 (English, with English and French summaries). http://dx.doi.org/10.1016/j.anihpb.2003.06.004. MR2096214.

[Run] V. RUNDE, Uniform continuity over locally compact quantum groups, J. Lond. Math. Soc. (2) 80 (2009), no. 1, 55-71. http://dx.doi.org/10.1112/jlms/jdp011. MR2520377.

[Sal] P. SALMI, Idempotent states on locally compact groups and quantum groups, Algebraic Methods in Functional Analysis, Oper. Theory Adv. Appl., vol. 233, Birkhäuser/Springer, Basel, 2014, pp. 155-170. http://dx.doi.org/10.1007/978-3-0348-0502-5_11. MR3203989.

$\left[\mathrm{SaS}_{1}\right]$ P. SALMI AND A. SKALSKI, Idempotent states on locally compact quantum groups, Q. J. Math. 63 (2012), no. 4, 1009-1032. http://dx.doi.org/10.1093/qmath/har023. MR2999995.

$\left[\mathrm{SaS}_{2}\right]$ - Actions of locally compact (quantum) groups on ternary rings of operators, their crossed products, and generalized Poisson boundaries, Kyoto J. Math. 57 (2017), no. 3, 667-691. http://dx.doi.org/10.1215/21562261-2017-0011. MR3685060.

[Tak] M. TAKESAKI, Theory of Operator Algebras. I, Reprint of the first (1979) edition; Operator Algebras and Non-commutative Geometry, 5, Encyclopaedia of Mathematical Sciences, vol. 124, Springer-Verlag, Berlin, 2002. MR1873025. 
[Tem] A. Tempelman, Ergodic Theorems for Group Actions: Informational and Thermodynamical Aspects, Translated and revised from the 1986 Russian original, Mathematics and its Applications, vol. 78, Kluwer Academic Publishers Group, Dordrecht, 1992. http://dx.doi.org/ 10.1007/978-94-017-1460-0. MR1172319.

[Vee] W. A. VEECH, Weakly almost periodic functions on semisimple Lie groups, Monatsh. Math. 88 (1979), no. 1, 55-68. http://dx.doi.org/10.1007/BF01305857. MR550072.

[Yau] S. T. YAU, Some function-theoretic properties of complete Riemannian manifold and their applications to geometry, Indiana Univ. Math. J. 25 (1976), no. 7, 659-670. http://dx. doi. org/10.1512/iumj.1976.25.25051. MR417452.

[Wi] S. Willard, General Topology, Addison-Wesley Publishing Co., Reading, Mass.-LondonDon Mills, Ont., 1970. MR0264581.

[Wil] G. A. WILlis, Probability measures on groups and some related ideals in group algebras, J. Funct. Anal. 92 (1990), no. 1, 202-263. http://dx.doi.org/10.1016/ 0022-1236 (90) 90075-V. MR1064694.

[Won] J. C. S. WONG, Invariant means on locally compact semigroups, Proc. Amer. Math. Soc. 31 (1972), 39-45. http://dx.doi.org/10.2307/2038508. MR289708.

MatThias NeUfang:

School of Mathematics \& Statistics

Carleton University

Ottawa, ON, K1S 5B

Canada

E-MAIL: mneufang@math.carleton.ca

PEKKA SALMI:

Department of Mathematical Sciences

University of Oulu

PL 3000, FI-90014 Oulun yliopisto

Finland

E-MAIL: pekka.salmi@iki.fi

ADAM SKALSKI:

Institute of Mathematics of the Polish Academy of Sciences

ul.Śniadeckich 8

00-656 Warszawa, Poland

E-MAIL: a.skalski@impan.pl

NICO SPRONK:

Department of Pure Mathematics

University of Waterloo

Waterloo, ON, N2L 3G1

Canada

E-MAIL:n.spronk@uwaterloo.ca

KEY WORDS AND PHRASES: Locally compact quantum group, convolution operators, contractive quantum measures, fixed points.

2000 Mathematics Subject Classification: 46L65 (43A05, 46L30, 60B15).

Received: July 19, 2019. 